\title{
$\Psi_{k}$ Scientific Highlight of the Month
}

No. 140

March 2018

\section{Spin-fluctuation and spin-relaxation effects of single adatoms from first principles}

\author{
Julen Ibañez-Azpiroz ${ }^{2}$, Manuel dos Santos Dias ${ }^{1}$, Stefan Blügel ${ }^{1}$, Samir Lounis ${ }^{1}$ \\ ${ }^{1}$ Peter Grünberg Institut and Institute for Advanced Simulation, Forschungszentrum Jülich $\mathcal{E}$ \\ JARA, 52425 Jülich, Germany \\ ${ }^{2}$ Centro de Física de Materiales, Universidad del País Vasco, 20018 San Sebastián, Spain
}

\begin{abstract}
Single adatoms offer an exceptional playground for studying magnetism and its associated dynamics at the atomic scale. Here we review recent results on single adatoms deposited on metallic substrates, based on time-dependent density functional theory. First we analyze quantum zero-point spin-fluctuations (ZPSF) as calculated from the fluctuationdissipation theorem, and show how they affect the magnetic stability by modifying the magnetic anisotropy energy. We also assess the impact of ZPSF in the limit of small hybridization to the substrate characteristic of semi-insulating substrates, connecting to recent experimental investigations where magnetic stability of a single adatom was achieved for the first time. Secondly, we inspect further the dynamics of single adatoms by considering the longitudinal and transverse spin-relaxation processes, whose time-scales are analyzed and related to the underlying electronic structure of both the adatom and the substrate. Thirdly, we analyze spin-fluctuation modes of paramagnetic adatoms, i.e., adatoms where the Stoner criterion for magnetism is almost fulfilled. Interestingly, such modes can develop well-defined peaks in the $\mathrm{meV}$ range, their main characteristics being determined by two fundamental electronic properties, namely the Stoner parameter and the density of states at the Fermi level. Furthermore, simulated inelastic scanning tunneling spectroscopy curves reveal that these spin-fluctuation modes can be triggered by tunneling electrons, opening up potential applications also for paramagnetic adatoms. Lastly, an overview of the outstanding issues and future directions is given.
\end{abstract}

Single adatoms deposited on surfaces have become a prominent playground where theory and experiment can explore hand-in-hand a large variety of physical phenomena ranging from spinexcitations $[1,2,3,4,5,6,7,8,9,10,11,12,13,14]$ to magnetic exchange interactions $[2,15$, $16,17,18,19,20]$, quantum spin decoherence [21, 22, 23], interplay between magnetism and superconductivity [24, 25, 26, 27, 28, 29, 30], or the Kondo effect [31, 32, 33, 34, 35, 36, 37], among many others. Virtually all these effects arise from the intricate interplay between the degrees of freedom of the adatom - charge, spin or orbital angular momentum - and the electron and phonon bath of the substrate, a subject of heavy and ongoing investigation. Noteworthily, 
magnetism plays a central role in fuelling the interest for these tiny objects, given that they represent the ultimate limit in the context of bit miniaturization in data storage devices. Therefore, understanding the above mentioned phenomena is of capital importance both for fundamental physics and for potential technological applications. In this context, the field has witnessed an enormous push forward after the advent of spin-polarized scanning tunnelling microscopy (STM) and inelastic electron tunnelling spectroscopy (IETS). These experimental techniques, occasionally used in combination with X-ray magnetic circular dichroism (XMCD) [38, 39] and electron paramagnetic resonance (EPR) [22], allow to monitor the dynamical regime by, e.g., measuring atomic spin-excitations [1, 3, 40, 8] and quasiparticle interferences [17], accessing spin-relaxation and decoherence times [41, 22, 19] and even resolving highly dynamical processes like the reading and writing of magnetic information [42].

From the theoretical point of view, early simulations based on density functional theory (DFT) [43, $44,45]$ served for boosting and creating a huge enthusiasm in the field by predicting gigantic local magnetic moments of diverse transition-metal (TM) adatoms, including $4 d$ and $5 d$ elements that are nominally nonmagnetic in bulk. In addition, the understanding and interpretation of features present in IETS experiments, such as the characteristic steps in the conductance spectrum [46], greatly benefited from studies based on quantum spin models, e.g., by showing the importance of inelastic excitation channels involved in the process (see, e.g., Refs. [47, 6, 48, 49, 50, 51]). In the last few years, the development of a real-space spin-dynamics technique within time-dependent DFT (TDDFT) [7, 52] has proven to be a powerful tool for characterizing the central dynamical quantity, namely the spin-excitation spectrum, which contains information about the exchange of energy and angular momentum with the substrate electrons that form the environment of the magnetic adatoms $[7,52]$. Importantly, a quantitative analysis of the role played by the spin-orbit coupling (SOC) in determining the resonance frequency of spin-excitations and their reaction to magnetic fields has been successfully given within this approach [53]. Going one step beyond, the use of many-body perturbation theory (MBPT) in connection with TDDFT has also made possible to establish a direct quantitative comparison to the $\mathrm{d} I / \mathrm{d} V$ (differential tunneling conductance) curves measured in IETS experiments [54].

One of the most fundamental requirements for a successful usage of single adatoms for technological purposes is the stability of its magnetic moment. This, in turn, depends crucially on the so-called magnetic anisotropy energy (MAE), an energy barrier generated by the SOC that protects and stabilizes the direction of the local magnetic moment against possible fluctuations of the spin, e.g., of thermal origin. Noteworthily, XMCD and IETS assert that several $3 d$ transition metal (TM) adatoms can possess a substantial MAE of few meV: examples include Fe and Co on $\mathrm{Pt}(111)$ [55, 9], $\mathrm{Fe}$ on $\mathrm{Cu}(111)$ [8] as well as on $\mathrm{Cu}_{2} \mathrm{Ni} / \mathrm{Cu}(111)$ [56] and $\mathrm{CuNi}$ [3], and $\mathrm{Co}$ on $\mathrm{MgO}(100)$ [57], among others. Strikingly, however, these large MAEs do not directly translate into an stable magnetic moment, given that the above mentioned systems behave as paramagnetic entities when measured by means of STM (see, e.g.,Refs. $[58,59,16]$ ), thus implying the existence of a mechanism that destroys the magnetic stability locally. Going one step further, the case of $4 d$ and $5 d$ adatoms is even more striking, given that they have so far exhibited no clear magnetic signal even when subjected to the static magnetic field of an XMCD experiment, in notorious disagreement with theoretical predictions [60]. Up to date, the only single adatom that has experimentally exhibited magnetic remanence is $\mathrm{Ho}$ on $\mathrm{MgO} / \mathrm{Ag}(100)$ [38, 42], i.e., a 
If rare-earth element with a very large local magnetic moment $\left(\sim 10 \mu_{\mathrm{B}}\right)$ deposited on a thin insulating layer.

In view of this rich scenario, a central question arises: what is the mechanism leading to the apparent instability of the magnetic moment of an adatom? Given that temperature effects are not the principal cause of the magnetic instability [58], one must turn to other destabilizing effects. Recent efforts to address this far-reaching issue have been mainly based on symmetry arguments applied to model Heisenberg Hamiltonians, whereby the switching probability of the magnetic moment is calculated by means of a master equation (see e.g. Refs. [61, 62, 63, 38]). These arguments apply to a well-localized magnetic moment of either integer or half-integer spin, which is justified in the weak coupling regime to the substrate. If the coupling is strong, as it is generally the case for metallic substrates, the orbitals responsible for the magnetic moment of an adatom hybridize with the surface conduction electrons, giving rise to an itinerant electron picture with fractional local magnetic moments (see, e.g., Refs. $[8,45,7,52,53]$ ). In the latter case, the proper description of the itinerant character is needed for addressing the subject of magnetic stability.

The present review focuses on the aforementioned strongly-hybridized metallic systems. In this context, zero-point spin-fluctuations (ZPSF) become a key dynamical aspect which are intimately connected to spin-excitations through the so-called fluctuation-dissipation theorem [64]. ZPSF are quantum fluctuations present even at absolute zero temperature that can crucially affect the magnetic properties of itinerant electron magnets. The importance of spin-fluctuations was first realized in the early studies of weak bulk ferromagnets [65, 66, 67, 68] and has subsequently been verified in the context of DFT and TDDFT too $[69,70]$. In this review, we consider the concept of ZPSF in single adatoms and quantify their impact on the magnetic stability of the series of $3 d$ and $4 d$ TM adatoms deposited on metallic substrates. We show that ZPSF are of the order of the local magnetic moment itself, an extremely large value that has deep effects on the MAE, which can be reduced by more than $50 \%$ with respect to the static value calculated by standard DFT. We further characterize the three fundamental ingredients that determine the magnitude of the ZPSF, namely the (i) local magnetic moment, (ii) SOC and (iii) electron-hole Stoner excitations. Based on our findings, we develop a simple diagram where the ZPSF of an arbitrary adatom are classified according to these ingredients, offering practical guidelines for achieving magnetic properties which are robust against fluctuations.

Apart from protecting the magnetic stability, in order to achieve the ultimate goal of a technologically useful single adatom it is mandatory to master the dynamical properties associated to its spin. For example, fast spin-dynamics can be useful when the goal is to transfer magnetic information from or to the adatom, while slow spin-dynamics are desirable if the aim is to store magnetic information. What are the typical time scales involved, and how does the environment affect them? Interestingly, the lifetime of an atomic spin-excitation can be experimentally accessed from the width of the step observed in IETS $\mathrm{d} I / \mathrm{d} V$ measurements, being typically of the order of picoseconds $[8,57,3,71]$. Throughout this review, we quantitatively connect this lifetime to electronic properties of adatoms and their hybridization with the substrate, showing that the single-particle spin-flip excitation channel is the main driving mechanism behind it. In tandem, recent experimental developments yielded another way to measure spin-relaxation times using an all-electrical pump-probe technique [41], which has been so far applied to adatoms 
deposited on insulating substrates [57, 22, 72]. Based on the ab initio perspective, here we show that, in order to successfully perform such experiments in metallic systems, a measuring scheme based on ultrafast techniques able to record relaxation times of the order of femtoseconds would be needed.

Although magnetic adatoms have by far attracted most of the interest in this field, nominally non-magnetic adatoms can also exhibit interesting fingerprints of magnetism and thus be potentially useful for technological purposes. This is the last main topic addressed in the present review, where we study what are the requirements for observing well-defined features in the spinexcitation spectrum of this type of adatoms, i.e., paramagnetic spin-excitations (PSE). These are the analogous of so-called paramagnons first proposed by Doniach in 1967 [73] and first measured in bulk Pd nearly 50 years later by Doubble and co-workers [74] (see also Ref. [75] for recent calculations). Upon reducing the dimensionality of the system, here we show that PSE can be strongly enhanced due to the modified interplay between the two fundamental electronic properties involved, namely the Stoner exchange interaction and the adatom's density of states (DOS) at the Fermi level. In addition, the ab initio analysis reveals that PSE are highly sensitive to externally applied magnetic fields and, furthermore, can exhibit a singular enhancement when the field approaches a critical regime. Finally, we assess the impact of PSE on the $\mathrm{d} I / \mathrm{d} V$ signal as measured in IETS experiments, identifying clear signatures of magnetic response that allow to distinguish these type of excitations from, e.g., phonons.

The review is organized as follows. In Sec. 1, we sketch the theoretical formalism used to calculate the quantities presented throughout the review. In Sec. 2 we begin by presenting the ground state properties of a set of $3 d$ and $4 d$ TM adatoms that will be the main focus of the review. In Sec. 3 the concept of ZPSF is presented as well as an analysis of its magnitude and its effect on inducing magnetic instability via the renormalization of the MAE barrier. In Section 4 , we focus on discussing atomic time scales for the relaxation time of the spin. Finally, in Sec. 5 we illustrate the concept of paramagnetic spin-fluctuation modes and possible means of probing it experimentally. Conclusions and outlook are given in Sec. 6, while appendices A and B contain derivations on the Bloch and Landau-Lifshitz-Gilbert models, respectively.

\section{Theoretical approach}

In this section we settle the theoretical background used throughout the review. For this, let us consider the linear response of a ferromagnetic system to an externally applied time-dependent perturbation,

$$
\delta \mathbf{m}(\mathbf{r} ; t)=\int \mathrm{d} \mathbf{r}^{\prime} \int \mathrm{d} t^{\prime} \chi\left(\mathbf{r}, \mathbf{r}^{\prime} ; t-t^{\prime}\right) \delta \mathbf{V}\left(\mathbf{r}^{\prime} ; t^{\prime}\right)
$$

Above, $\delta \mathbf{m}=\left(\delta m_{x}, \delta m_{y}, \delta m_{z}, \delta n\right)$ and $\delta \mathbf{V}=\left(\delta B_{x}, \delta B_{y}, \delta B_{z}, \delta V\right)$, with $\delta m_{i}$ and $\delta B_{i}$ respectively the components of the vector spin density and external magnetic field, while $\delta n$ and $\delta V$ are the charge density and external scalar potential, respectively. Working in frequency space and defining atomic-like quantities by integrating out the spatial dependence over atomic sites [52], the above expression takes the simplified form

$$
\delta \mathbf{m}(\omega)=\chi(\omega) \delta \mathbf{V}(\omega) .
$$


For a single magnetic atom, the quantity $\chi$ in the above equations is a $4 \times 4$ tensor that couples in general all components of the spin and charge responses with each other. If SOC is weak, however, the full response decouples into a longitudinal and transverse part [76]. This approximation is justified for the systems investigated in this review since the off-diagonal sectors of the susceptibility tensor are small in comparison to the diagonal ones. Then, assuming that the perturbation is purely of magnetic origin (i.e. $\delta V=0$ ), the change of the spin magnetic moment length is described by

$$
\delta m_{z}(\omega)=\chi_{\|}(\omega) \delta B_{z}(\omega)
$$

Above, $\chi_{\|}(\omega)$ denotes the longitudinal spin-susceptibility. This quantity is determined by excitations between electrons with same spin state, given that it involves the Pauli matrix $\sigma_{z}$ that is diagonal in spin basis [77]. On the other hand, the change of the transverse spin components can be compactly described using the circular combinations $m_{ \pm}=m_{x} \pm \mathrm{i} m_{y}$ and $B_{ \pm}=B_{x} \pm \mathrm{i} B_{y}$, yielding for the + component

$$
\delta m_{+}(\omega)=\chi_{ \pm}(\omega) \delta B_{+}(\omega)
$$

Above, $\chi_{ \pm}(\omega)$ denotes the transverse spin-susceptibility which, contrary to $\chi_{\|}(\omega)$, is determined by transitions that flip the spin state of the electrons due to the transverse Pauli spin matrices involved, which are off-diagonal in spin space $[7,52,53]$. We note that the full transverse response is given by

$$
\chi_{\perp}(\omega) \equiv 2\left(\chi_{ \pm}(\omega)+\chi_{\mp}(\omega)\right)
$$

The spin-susceptibility is closely connected to the ZPSF via the so-called fluctuation-dissipation theorem [64]. This theorem relates the variance of the spin-fluctuations along a given direction, $\xi_{i}^{2}$, to the imaginary part of the corresponding spin-susceptibility, $\operatorname{Im} \chi_{i}(\omega)$,

$$
\xi_{i}^{2}=-\frac{1}{\pi} \int_{0}^{\infty} \mathrm{d} \omega \operatorname{Im} \chi_{i}(\omega)
$$

with $i=\|, \perp$.

The calculation of the ZPSF requires therefore a realistic and accurate description of the spinsusceptibility of the adatom. This can be achieved by employing the time-dependent generalization of DFT, namely TDDFT [78, 79]. The general expression for the adatom's enhanced spin-susceptibility within the TDDFT framework is given by $[7,52,53,80,54,81]$

$$
\chi_{i}(\omega)=\frac{\chi_{i}^{\mathrm{KS}}(\omega)}{1-U_{i} \chi_{i}^{\mathrm{KS}}(\omega)},
$$

with $i=\|, \pm, \mp$. Above, $\chi_{i}^{\mathrm{KS}}(\omega)$ is the bare $\mathrm{KS}$ spin-susceptibility, i.e. the standard response function in linear response theory. For the analysis provided in this review, we calculate the different components of $\chi_{i}^{\mathrm{KS}}(\omega)$ using the Korringa-Kohn-Rostoker Green function (KKRGF) method employing the atomic sphere approximation with full charge density [82]. For details on the formalism, we refer the reader to Refs. [52, 53]

Finally, we note that $U_{i}$ in Eq. 7 denotes the interaction kernel, which in this work is treated in the adiabatic local spin-density approximation [83]. In the longitudinal case, the kernel 

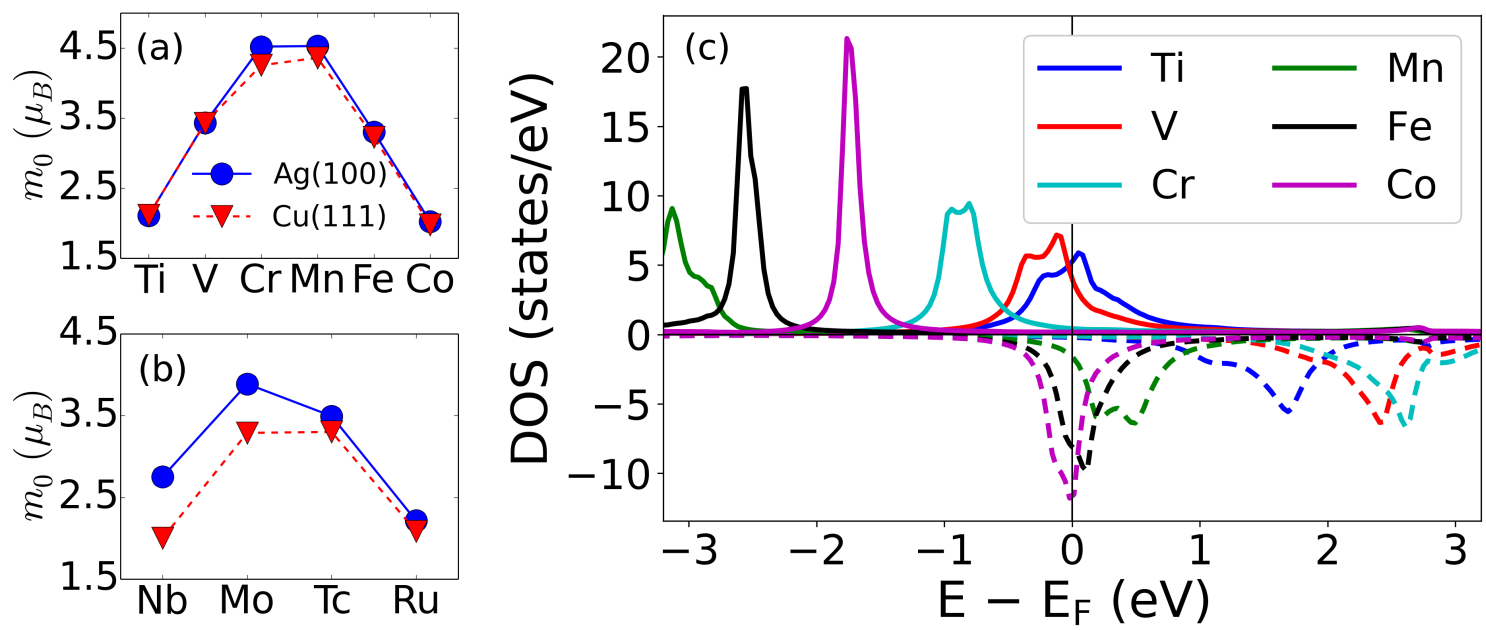

Figure 1: Calculated ground state electronic properties of single adatoms. (a) Spin magnetic moments for several $3 d$ transition metal adatoms deposited on $\mathrm{Ag}(100)$ and $\mathrm{Cu}(111)$ denoted by circles (blue) and triangles (red), respectively. (b) Same as (a) but for $4 d$ adatoms. (c) Atom-projected total electronic DOS for $3 d$ adatoms deposited on $\operatorname{Ag}(100)$. The majority and minority spin channels are denoted by solid (positive) and dashed (negative) lines, respectively. The vertical line denotes the Fermi level.

$U_{\|}$contains two contributions, namely the Coulomb term as well as the exchange-correlation (XC) term responsible for the many-body effects [70, 84]. In the transversal case, the Coulomb term drops out leaving only the XC term. Importantly, the transverse kernel $U_{ \pm}=U_{\mp}$ can be connected to the magnetic moment $m_{0}$ and the XC magnetic field $B_{\mathrm{xc}}$ via sum-rules that allow an accurate description of the dynamical regime: for details, we refer the reader to Refs. $[7,52,53]$.

\section{Preliminaries: ground state properties of $3 d$ and $4 d$ magnetic TM single adatoms deposited on metallic substrates}

The main focus of this review is a set of $3 d$ and $4 d$ adatoms deposited on the metallic $\operatorname{Ag}(100)$ and $\mathrm{Cu}(111)$ substrates. Before tackling their dynamical properties, we first analyze two ground state quantities, namely the spin magnetic moment, denoted as $m_{0}$, and the electronic DOS. Let us begin with Fig. 1(a) and 1(b), where the calculated $m_{0}$ is depicted. This figure shows that all the considered adatoms develop large magnetic moments of more than $2 \mu_{\mathrm{B}}$. Furthermore, $m_{0}$ acquires non-integer values, indicating the itinerant character of the adatom's $d$-electrons induced by the hybridization with the electrons of the metallic substrate. This feature is confirmed by the DOS, which is displayed in Fig. 1(c) for the specific case of $3 d$ adatoms deposited on $\operatorname{Ag}(100)$. This figure shows that the $d$-state peaks, so-called virtual bound states, are substantially broadened (between $\sim 0.1 \mathrm{eV}$ and $\sim 1 \mathrm{eV}$ depending on the adatom), a well-known consequence of hybridization with the substrate [44, 8, 53]. Another property evidenced by Figs. 1(a,b) is that the first of the atomic Hund's rules is closely fulfilled, i.e. the half filled $d$-shell elements develop largest magnetic moments, case of $\mathrm{Cr}$ and $\mathrm{Mn}$ for $3 d$, Mo and Tc for $4 d$. Finally, Figs. 1(a,b) show that the choice of metallic substrate and surface orientation does 


\begin{tabular}{c|c|c|c|c|c|c||c|c|c|c|c}
\hline & $\mathrm{Ti}$ & $\mathrm{V}$ & $\mathrm{Cr}$ & $\mathrm{Mn}$ & $\mathrm{Fe}$ & $\mathrm{Co}$ & $\mathrm{Zr}$ & $\mathrm{Nb}$ & $\mathrm{Mo}$ & $\mathrm{Tc}$ & $\mathrm{Ru}$ \\
\hline$\sqrt{\xi_{\|}^{2}}[\mathrm{Ag}(100)]$ & 0.09 & 0.11 & 0.06 & 0.07 & 0.14 & 0.18 & 0.21 & 0.16 & 0.22 & 0.16 & 0.34 \\
\hline$\sqrt{\xi_{\perp}^{2}}[\mathrm{Ag}(100)]$ & 2.05 & 2.50 & 3.21 & 3.25 & 3.16 & 1.63 & 0.92 & 2.41 & 2.84 & 3.51 & 1.88 \\
\hline \hline$\sqrt{\xi_{\|}^{2}}[\mathrm{Cu}(111)]$ & 0.12 & 0.14 & 0.12 & 0.10 & 0.08 & 0.14 & - & 0.36 & 0.36 & 0.38 & 0.24 \\
\hline$\sqrt{\xi_{\perp}^{2}}[\mathrm{Cu}(111)]$ & 2.49 & 2.80 & 2.31 & 2.56 & 3.02 & 2.57 & - & 2.31 & 2.56 & 2.70 & 2.27 \\
\hline
\end{tabular}

Table 1: Calculated longitudinal and transverse contribution to the ZPSF (units of $\mu_{\mathrm{B}}$ ).

not substantially affect the spin magnetic moment of the adatom, impliying that the substrate's symmetry plays only a minor role in this context. We note that these ground state properties are consistent with the original works by Dederichs and co-workers [43, 44, 45], as well as with more recent studies $[7,52,53,80,54,81]$.

\section{Zero-point spin-fluctuations}

In this section we analyze the ZPSF (Eq. 6) associated to the magnetic single adatoms presented in Sec. 2. Two fundamentally different contributions can be distinguished: the longitudinal $\left(\xi_{\|}^{2}\right)$ and transverse $\left(\xi_{\perp}^{2}\right)$ components of the ZPSF. In Table 3 we have listed the calculated magnitude of both quantities. Importantly, these values show that the longitudinal contribution is approximately an order of magnitude smaller than the transverse one: the longitudinal component reaches a maximum of $\sqrt{\xi_{\|}^{2}}=0.38 \mu_{\mathrm{B}}$ for Mo on $\operatorname{Ag}(100)$, while $\sqrt{\xi_{\perp}^{2}}>1 \mu_{\mathrm{B}}$ for virtually all the adatoms. This feature reflects the fact that it is much easier to alter the direction of the magnetic moment than its size. Thus, given that the major contribution to the fluctuations comes from the transverse component, in the following we will focus on its analysis and its effects on the magnetic stability.

\subsection{Connection to spin-excitation spectra}

The integrand of Eq. 6 defining the ZPSF is composed by the imaginary part of the enhanced spin-susceptibility, a quantity that displays so-called spin-excitations associated to the damped precessional motion of the spin-moment $[7,52,53]$. Calculated transverse spin-excitation and spin-fluctuation spectra for TM adatoms deposited on $\mathrm{Ag}(100)$ are shown in Fig. 2. Let us first focus on Figs. 2(a,c), which display $\operatorname{Im} \chi_{\perp}(\omega)$ for $3 d$ and $4 d$ adatoms, respectively. These figures reveal the existence of a large peak in the meV range for all adatoms, corresponding to a spin-excitation. Note that the associated resonance frequency is dependent on the MAE, which is in turn settled by the SOC [53]. The largest resonance frequency of $\sim 6 \mathrm{meV}$ pertains to the Fe adatom, while the rest of adatoms display the peak at $\lesssim 4 \mathrm{meV}$; in the particular cases of $\mathrm{Ti}, \mathrm{V}, \mathrm{Mn}, \mathrm{Cr}$ and Mo, we find the spin-excitation at extremely small frequencies, $\lesssim 0.5 \mathrm{meV}$, impliying that the net effect of SOC is very weak in these adatoms. Figs. 2(a,c) display yet another feature, namely the width of the spin-excitation peak, which is linked to the amount of electron-hole Stoner excitations near the Fermi level [80]: $\mathrm{Ru}$ and, to some extent also Nb and Tc, display large widths as compared to the rest of adatoms, in particular those that peak 

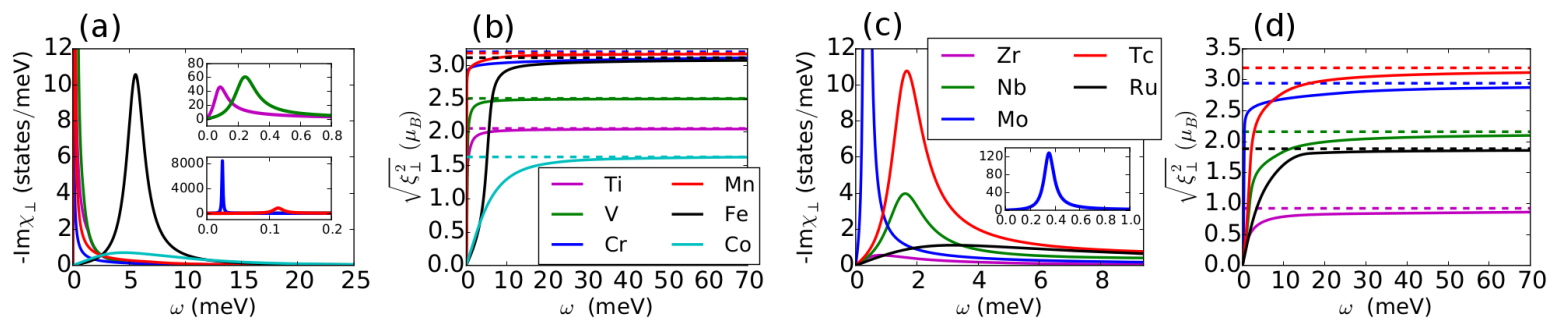

Figure 2: Calculated spin-excitation spectra and ZPSF. (a) and (c) Density of transverse spinexcitations as given by $\operatorname{Im} \chi_{\perp}(\omega)$ for selected $3 d$ and $3 d$ adatoms on $\operatorname{Ag}(100)$, respectively. Insets address different frequency regions where various resonance frequencies are located. (b) and (d) Calculated magnitude of the mean value of transverse ZPSF for the adatoms considered in (a) and (c), respectively. Solid lines depict the evolution of the value as a function of the upper boundary of the integral of Eq. 6, while the horizontal dashed lines represent the converged value.

below $1 \mathrm{meV}$.

We next turn to the magnitude of the transverse ZPSF (Eq. 6), as displayed in Figs. 2(b,d) for $3 d$ and $4 d$ adatoms on $\operatorname{Ag}(100)$, respectively; the solid lines depict the evolution of the mean value, $\sqrt{\xi_{\perp}^{2}}$, as a function of the upper boundary of the frequency integral, whereas the converged value is marked by the horizontal dashed lines. As already pointed in the discussion of Table $3, \sqrt{\xi_{\perp}^{2}}$ is of the order of the Bohr magneton; the elements with largest values are $\mathrm{Cr}, \mathrm{Mn}, \mathrm{Fe}$ and Tc, with $\sqrt{\xi_{\perp}^{2}} \sim 3 \mu_{\mathrm{B}}$, whereas in $\mathrm{Zr}$ and Co this value is reduced by more than $50 \%$. As the calculations demonstrate, the main contribution to the integral of Eq. 6 comes from the spin-excitation peak in the meV region, which represents between $70 \%$ and $\sim 100 \%$ of the total depending on the adatom.

Figs. 3(a) and 3(b) show an instructive comparison between the magnitude of the transverse ZPSF and the local magnetic moments of the adatoms. These figures demonstrate that $\sqrt{\xi_{\perp}^{2}}$ (blue stars) is always an appreciable fraction of $m_{0}$ (blue circles); one finds $\sqrt{\xi_{\perp}^{2}} \sim m_{0} / 2$ for $\mathrm{Mn}$ and $\mathrm{Cr}$, while $\sqrt{\xi_{\perp}^{2}} \sim m_{0}$ for $\mathrm{Ti}, \mathrm{Fe}, \mathrm{Zr}, \mathrm{Nb}$ and $\mathrm{Ru}$, revealing that the latter suffer from strong deviations of the direction of the local magnetic moment. We also note that both $m_{0}$ and $\sqrt{\xi_{\perp}^{2}}$ follow the evolution predicted by Hund's rules, i.e. adatoms with nearly half-filled $d$-shells have largest values: $\mathrm{Cr}$ and $\mathrm{Mn}$ among $3 d$, and Mo and Tc among $4 d$. This trend is clearly fulfilled in the case of the magnetic moment as already mentioned in Sec. 2, while the evolution of the fluctuations shows some exceptions like Fe and Tc. Noteworthily, a similar relationship between magnetic moment and fluctuations has been very recently found also for bulk transition metals [85]. In addition, Fig. 3 also reveals that the spin-fluctuation-to-magnetization ratio (SFMR) is generally larger in $3 d$ adatoms than in $4 d$. As a final remark, let us note that Mo, which is the only $4 d$ adatom deposited on $\operatorname{Ag}(100)$ that shows an experimentally measurable magnetic signal [86], has by far the lowest SFMR among $4 d$ elements.

Figs. 3(a) and 3(b) also display an extension of the analysis to the same set of adatoms deposited on $\mathrm{Cu}(111)$, which exhibit essentially the same features as on $\mathrm{Ag}(100)$. This again indicates that symmetry plays a minor role in these largely hybridized metallic systems with strong itinerant 

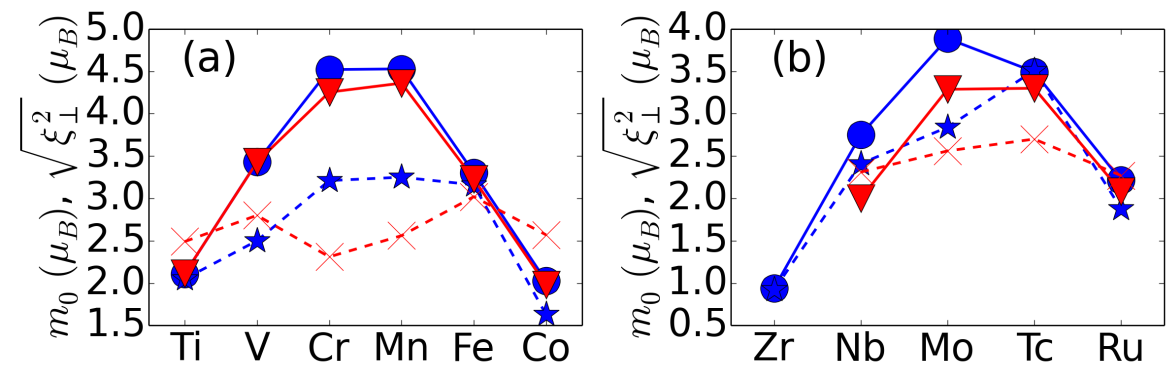

Figure 3: Comparison of local magnetic moment and mean value of the transverse ZPSF. (a) $3 d$ adatoms and (b) $4 d$ adatoms. As in Figs. 1(a,b), blue circles and red triangles denote $m_{0}$ for adatoms deposited on $\mathrm{Ag}(100)$ and $\mathrm{Cu}(111)$, respectively. In addition, blue stars and red crosses denote $\sqrt{\xi_{\perp}^{2}}$ for adatoms deposited on $\mathrm{Ag}(100)$ and $\mathrm{Cu}(111)$, respectively.

character. The only noteworthy difference is that the SFMR of Cr and Mn is somewhat lower than what is expected from the trend of Fig. 3; we will discuss the origin of this feature shortly. Let us also note that Co adatoms show a very large SFMR in both $\mathrm{Ag}(100)$ and $\mathrm{Cu}(111)$ (see Fig. 3). Incidentally, Co is known to behave as a Kondo system when deposited on various metallic substrates $[87,88,89]$. Since both ZPSF and the Kondo effect are driven by spin-flip excitations with substrate electrons (see discussion below and Fig. 4), it is tempting to associate both effects. However, only a more advanced theory capable of describing such many-body effects can prove this hypothesis.

\subsection{Interpretation in terms of the Landau-Lifshitz-Gilbert model}

In the following, we turn to identify the fundamental factors that determine the magnitude of the transverse ZPSF. For this purpose, we consider the Landau-Lifshitz-Gilbert (LLG) equation [90]. The LLG is widely employed for characterizing the spin-dynamics of macroscopic magnetic systems, and more recently also for microscopic systems $[80,53]$ as it allows to accurately reproduce the $a b$ initio calculations by extracting the relevant parameters (see also Ref. [91] for a derivation of the quantum counterpart of the LLG equation). The details of the model are worked out in Appendix B. Here, let us recall that within the LLG model, the imaginary part of the transverse spin-susceptibility takes the form of an skewed Lorentzian, i.e.

$$
\operatorname{Im} \chi_{ \pm}^{\mathrm{LLG}}(\omega)=\frac{M \gamma}{2\left(1+\eta^{2}\right)} \cdot \frac{\eta \omega}{\left(\omega-\omega_{0}\right)^{2}+\left(\eta \omega_{0}\right)^{2}} .
$$

The parameters entering this model are the Gilbert damping, $\eta$, which determines the width of the spin-excitation peak and is dominated by Stoner excitations, and the resonance frequency, $\omega_{\text {res }}=\omega_{0} \sqrt{1+\eta^{2}}=\gamma B_{\text {eff }} / \sqrt{1+\eta^{2}}$, where $\gamma$ is the gyromagnetic ratio and $B_{\text {eff }}$ an effective magnetic field whose magnitude is determined by the strength of SOC (and of the external field, if considered); a detailed discussion can be found in, e.g., Ref. [53].

The integral of Eq. 6 can be calculated analytically within the LLG model and yields immediately the expression for the transverse ZPSF:

$$
\xi_{\mathrm{LLG}}^{2}=\frac{M \gamma}{\pi\left(1+\eta^{2}\right)}\left(\frac{\eta}{2} \log \frac{\left(x^{2}+\eta^{-2}+1\right)^{2}-\left(2 x \eta^{-1}\right)^{2}}{\left(\eta^{-2}+1\right)^{2}}+\vartheta(x, \eta)\right) .
$$




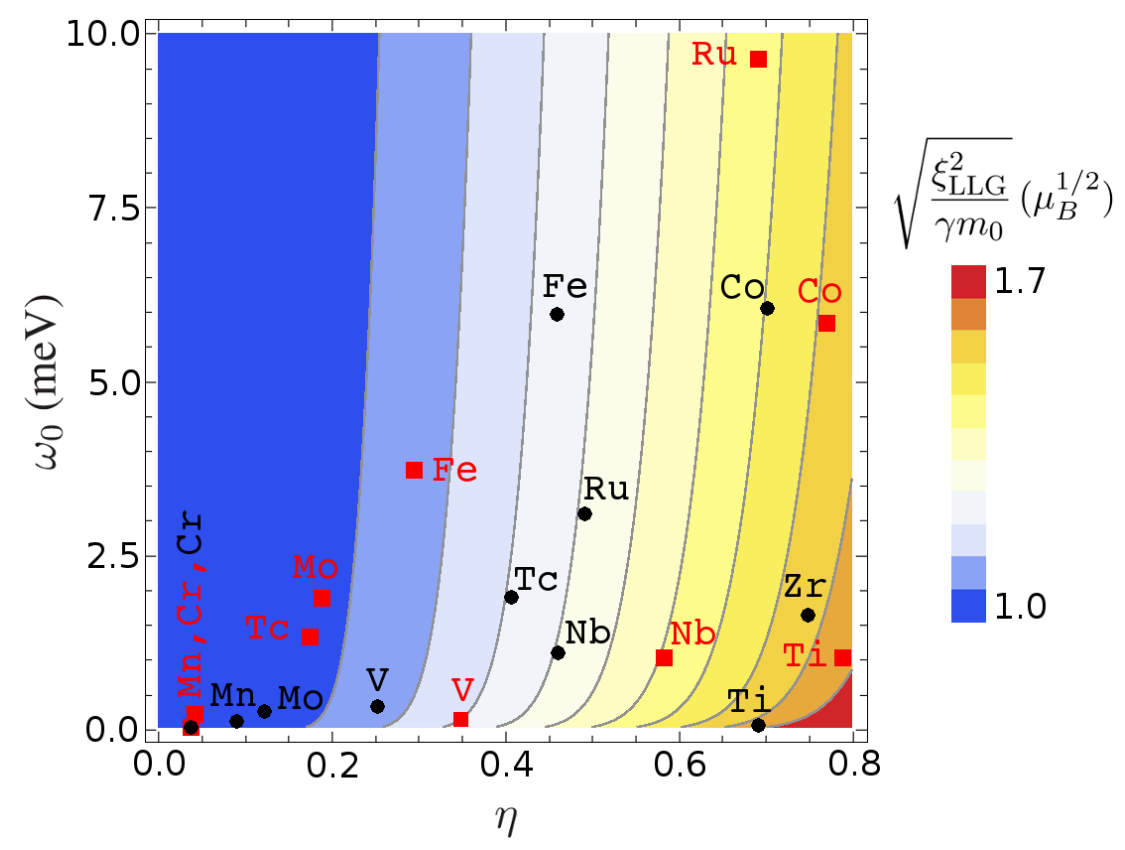

Figure 4: The background shows a 2D plot of the SFMR as a function of the damping and the resonance frequency in the LLG model (Eq. 9). Circles (black) and squares (red) denote various adatoms deposited on $\mathrm{Ag}(100)$ and $\mathrm{Cu}(111)$, respectively, where the parameters $\eta$ and $\omega_{0}$ (see discussion of Eq. 8) have been extracted from a fit to the ab initio spin-susceptibility.

Above, $\vartheta(x, \eta)=\arctan \left(x-\eta^{-1}\right)-\arctan \left(x+\eta^{-1}\right)+2 \arctan \eta^{-1}$ and $x=\omega_{c} / \eta \omega_{0}$, with $\omega_{c}$ a cutoff frequency to be converged.

Eq. 9 provides an interpretation for the magnitude of the fluctuations in terms of the physical parameters of the LLG model, which are in turn related to the electronic structure of the adatoms. In particular, three major ingredients come into play: $m_{0}, \omega_{0}$ and $\eta$. First of all, Eq. 9 reveals that $\sqrt{\xi_{\mathrm{LLG}}^{2}}$ is proportional to $\sqrt{m_{0}}$, which already accounts for the rough proportionality displayed by the ab initio calculations (see Fig. 3). Moreover, this relationship further indicates that fluctuations are relatively weaker for adatoms with large magnetic moments, namely the nearly half-filled $d$-shell elements. Interestingly, the simple relationship of Eq. 9 allows to analyze the SFMR, $\sqrt{\xi_{\mathrm{LLG}}^{2} / \gamma m_{0}}$, as a function of $\eta$ and $\omega_{0}$ by setting the standard value for the gyromagnetic ratio $\gamma=2$; the map obtained by doing so is shown in the background of Fig. 4 . This figure evidences that the SFMR is very much enhanced by the damping since $\sqrt{\xi_{\text {LLG }}^{2} / \gamma m_{0}}$ varies by almost $70 \%$ in the range of values considered for $\eta$. In contrast, Fig. 4 shows that the transverse ZPSF are reduced for large resonance frequencies, but the induced variation is much less important than that of the damping. In order to quantitatively connect these features to $a b$ initio calculations, we have extracted the parameters $\eta$ and $\omega_{0}$ by fitting $\operatorname{Im} \chi_{ \pm}^{\mathrm{LLG}}(\omega)$ to the calculated spin-susceptibility (e.g., Figs. 2(a,c)) for all adatoms. In this way, we locate the position of each adatom on the map of Fig. 4, as depicted by the circles and squares for the case of the $\mathrm{Cu}(111)$ and $\mathrm{Ag}(100)$ substrates, respectively. The resulting distribution evidences that the origin of the large SFMR found in the case of $\mathrm{Ti}, \mathrm{Co}, \mathrm{Ru}$ and $\mathrm{Nb}$ on both substrates, as well as $\mathrm{Fe}$ and $\mathrm{Zr}$ on $\mathrm{Ag}(100)$, is mainly due to the large damping factors of these adatoms, $\eta \gtrsim 0.5$, while this tendency is only slightly modified by the position of the resonance frequency. 
We note that a remarkable feature is revealed when $\eta$ tends to zero, i.e.,

$$
\lim _{\eta \rightarrow 0} \sqrt{\frac{\xi_{\mathrm{LLG}}^{2}}{\gamma m_{0}}}=1 \mu_{\mathrm{B}}^{1 / 2} \Rightarrow \lim _{\eta \rightarrow 0} \sqrt{\xi_{\mathrm{LLG}}^{2}}=\sqrt{\gamma m_{0}} .
$$

The expression above shows that the transverse ZPSF remain finite even in the extreme case of a spin-excitation with vanishing width. This intrinsic contribution is required by Heisenberg's uncertainty principle, since different spin-operators do not commute with each other [92], thus setting a lower boundary for the fluctuations. This is indeed the case found for $\mathrm{Mn}, \mathrm{Cr}$ and $\mathrm{Mo}$ adatoms, which, despite their small resonance frequency, their tiny damping $(\eta \lesssim 0.05)$ makes

$\sqrt{\xi_{\mathrm{LLG}}^{2} / \gamma m_{0}}$ approach the intrinsic minimum value for these elements. Interestingly, we note that Eq. 10 represents a sensible limit to give a rough estimate for the ZPSF of adatoms deposited on insulating and semiconducting substrates, since the substrate hybridization giving rise to the damping tends to be far smaller on those type of substrates as compared to the metallic ones considered in this review. Let us illustrate this notion by considering the important example of a Ho atom deposited on ultrathin $\mathrm{MgO}(100)$ layers grown on $\mathrm{Ag}(100)$, which experimentally exhibits a stable magnetic moment for time-periods larger than $10^{3} \mathrm{~s}$ [38]. From Hund's rules the Ho adatom should have a magnetic moment of $10 \mu_{\mathrm{B}}$, as found experimentally [42]. Under this assumption, a quick estimate using Eq. 10 reveals that the magnitude of the ZPSF in Ho should be $\sim 4.5 \mu_{\mathrm{B}}$. This, in turn, means that the resulting SFMR is nearly a factor of 2 smaller than in the elements with lowest SFMR studied in this review (see Eq. 13 and Fig. 5), implying that the relative impact of the quantum spin-fluctuations for $\mathrm{Ho}$ on $\mathrm{MgO} / \mathrm{Ag}(100)$ is particularly weak.

\subsection{Impact on the magnetic anisotropy energy}

As a last step in the analysis of this section devoted to spin-fluctuations, we turn now to analyze the effects of the transverse ZPSF on the magnetic stability of the adatoms. For this purpose, we estimate how the fluctuations affect the MAE, the quantity defining the height of the energy barrier when the magnetic moment is made to rotate away from its easy axis. We note that the MAE is closely connected to the resonance frequency of the spin-excitations (see, e.g., Ref. [53]) that give rise to the primary contribution to the transverse ZPSF; most importantly, the energy scale of both quantities is the same (meV).

We consider the following expression for the MAE for uniaxial systems,

$$
E_{a}(\theta)=\mathrm{K} \frac{\left(\mathbf{m} \cdot \hat{\mathbf{e}}_{z}\right)^{2}}{\mathbf{m}^{2}}=\mathrm{K} \cos ^{2} \theta
$$

where $\mathrm{K}$ is the so-called anisotropy constant. As a consequence, the energy barrier between the magnetic moment pointing along the $z$ axis $(\theta=0)$ and a perpendicular axis $(\theta=\pi / 2)$ is given by $E_{a}(\theta=0)-E_{a}(\theta=\pi / 2)=\mathrm{K}$, as it is schematically illustrated in Fig. 5(a). In the spirit of the spin-fluctuation theory of Moriya [65], we now let magnetic moment fluctuate around its equilibrium value, i.e. $\mathbf{m}^{2} \rightarrow\left(m_{0} \hat{\mathbf{e}}_{z}+\sum_{\perp} \boldsymbol{\xi}_{\perp}\right)^{2}$. Introducing this term into the definition of $E_{a}(\theta)$, we obtain a renormalized expression for the MAE, i.e.

$$
E_{a}\left(\theta, \xi_{\perp}^{2}\right)=\frac{\mathrm{K}\left(m_{0}^{2} \cos ^{2} \theta+\xi_{\perp}^{2} \sin ^{2} \theta\right)}{m_{0}^{2}+2 \xi_{\perp}^{2}} .
$$


(a)

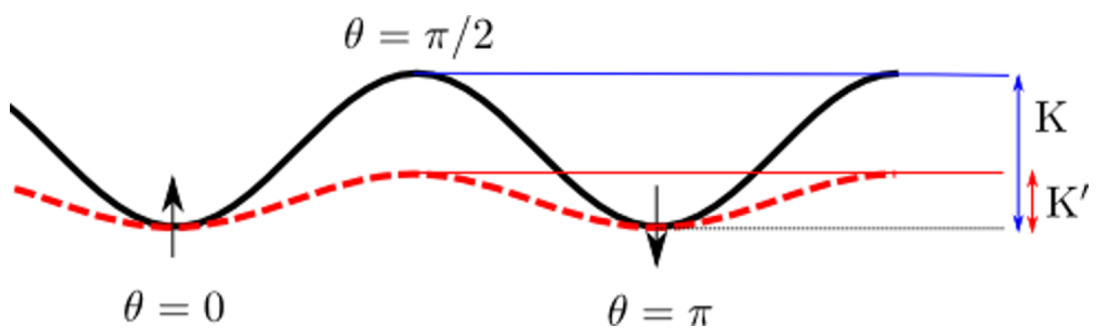

(b)

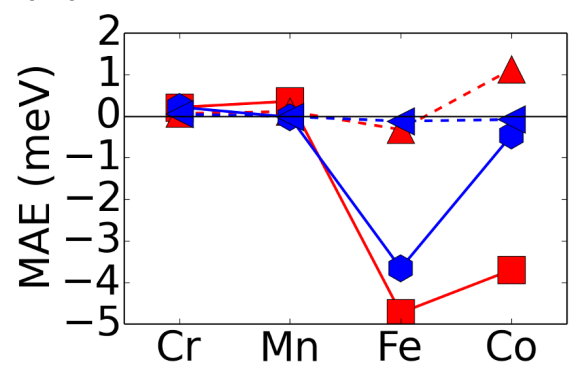

(c)

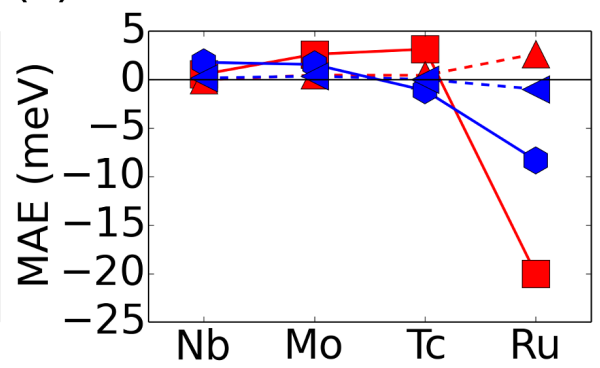

Figure 5: (a) Schematic illustration of the renormalization of the MAE induced by the transverse ZPSF. $\mathrm{K}$ and $\mathrm{K}^{\prime}$ respectively represent the static and renormalized anisotropy constant (see Eq. 13). (b) and (c) Calculated anisotropy constants for $3 d$ and $4 d$ adatoms, respectively. Blue hexagons and leftward triangles denote $\mathrm{K}$ and $\mathrm{K}^{\prime}$ for adatoms on $\mathrm{Ag}(100)$, respectively, while red squares and upward triangles denote $\mathrm{K}$ and $\mathrm{K}^{\prime}$ for adatoms on $\mathrm{Cu}(111)$, respectively.

In practice, this implies that the energy barrier gets effectively reduced by the transverse ZPSF,

$$
E_{a}\left(\theta=0, \xi_{\perp}^{2}\right)-E_{a}\left(\theta=\pi / 2, \xi_{\perp}^{2}\right)=\mathrm{K}\left(1-\frac{3 \xi_{\perp}^{2}}{m_{0}^{2}+2 \xi_{\perp}^{2}}\right) \equiv \mathrm{K}^{\prime} .
$$

The above is characterized by a modified anisotropy constant $\mathrm{K}^{\prime}$, also illustrated in Fig. 5(a).

In Figs. 5(b) and 5(c) we show the calculated values for the anisotropy constant, K, as well as for the reduced one, $\mathrm{K}^{\prime}$ : the former has been evaluated by band energy differences following the magnetic force theorem [93], while the latter has been calculated using the values of the magnetic moments and transverse ZPSF of the adatoms. These figures show a large and generalized reduction of the MAE, which goes beyond estimates that do not take into account fluctuation effects [53]. In cases where the ZPSF are larger than $m_{0}$ (mostly $4 d$ adatoms), the magnetic moment direction becomes destabilized, as reflected by the sign change in the renormalized MAE. Let us pay special attention to the well-studied case of $\mathrm{Fe}$ on $\mathrm{Cu}(111)$, where the MAE is reduced from $\mathrm{K}=-4.73 \mathrm{meV}$ to $\mathrm{K}^{\prime}=-0.32 \mathrm{meV}$. Interestingly, this means that ZPSF considerably remedy the disagreement with the experimental MAE, which is nearly $\sim-1 \mathrm{meV}[8,94]$. More importantly, this example shows that the renormalization of the MAE due to the ZPSF has the correct order of magnitude. Focusing next on the $4 d$ elements $\mathrm{Ru}$ and $\mathrm{Nb}$ on $\mathrm{Ag}(100)$, we find that the reduction of the MAE predicted by the calculations is notably large, i.e. $\gtrsim 90 \%$. It is noteworthy that these two cases have been thoroughly studied experimentally, which have concluded that there is no sizable magnetic signal in the systems [60, 95]. This suggests that the ZPSF might also play a major role in the experimentally observed absence of stable magnetism. 


\section{Spin-relaxation times}

While the analysis of Sec. 3 deals with the magnitude of the fluctuations, this section instead focuses on the time scales. For this purpose, we consider two particular relaxation processes of magnetic single adatoms, namely the longitudinal and transverse spin-relaxations characterized by the relaxation times $T_{\|}$and $T_{\perp}$, respectively. Physically, $T_{\|}$characterizes the relaxation of the size of the adatom's spin magnetic moment while $T_{\perp}$ describes its damped precessional motion. In the following, we analyze each of this processes separately.

\subsection{Longitudinal component}

In essence, the longitudinal spin-susceptibility $\chi_{\|}(\omega)$ (see Eq. 7) describes the ability of the system to continuously modify the size of its magnetic moment by an externally applied timedependent magnetic perturbation along the magnetization direction. The dynamics of this process can be phenomenologically studied in terms of the longitudinal Bloch equation, which yields the following form for the enhanced spin-susceptibility [96] (see Appendix A),

$$
\chi^{\mathrm{Bl}}(\omega)=\frac{\chi_{0}^{\mathrm{Bl}}}{1-\mathrm{i} \omega T_{\|}} .
$$

Above, $\chi_{0}^{\mathrm{Bl}}$ denotes a static spin-susceptibility, while $T_{\|}$corresponds to the longitudinal relaxation time mentioned in the introduction above. The aim is to establish a direct comparison between Eqs. 7 and 14. For this purpose, let us use the first-order Taylor expansion of the KS spin-susceptibility [77]

$$
\chi_{\|}^{\mathrm{KS}}(\omega) \simeq \rho_{\mathrm{F}}-\mathrm{i} n_{\mathrm{e}-\mathrm{h}} \omega,
$$

with $\rho_{\mathrm{F}}=\rho_{\mathrm{F}, \uparrow}+\rho_{\mathrm{F}, \downarrow}$ the DOS at the Fermi level and $n_{\mathrm{e}-\mathrm{h}}=\pi\left(\rho_{\mathrm{F}, \uparrow}^{2}+\rho_{\mathrm{F}, \downarrow}^{2}\right) / 2$ the density of electron-hole excitations of the same spin channel. By inserting $\chi_{\|}^{\mathrm{KS}}(\omega)$ of Eq. 15 into Eq. 7, $\chi_{\|}(\omega)$ acquires a functional form in $\omega$ equal to that of $\chi^{\mathrm{Bl}}(\omega)$ in Eq. 14. This then allows to obtain an expression for the longitudinal relaxation time in terms of basic electronic properties (see Appendix A for details):

$$
T_{\|}=\frac{U_{\|} n_{\mathrm{e}-\mathrm{h}}}{U_{\|} \rho_{\mathrm{F}}-1} .
$$

The above expression shows that the longitudinal relaxation time is mostly settled by the magnitude of electron-hole excitations weighted by the XC kernel (see the numerator of Eq. 16); this product is of order $1 / \mathrm{eV}$, hence settling the overall time scale of $T_{\|}$as fs. Furthermore, Eq. 16 shows that $T_{\|}$diverges as $U_{\|} \rho_{\mathrm{F}} \rightarrow 1$ (see the unitless denominator in the equation) and hence its magnitude can be strongly modified in this limit, i.e. as the system approaches the magnetic transition point. In the following, we first focus on quantitatively analyzing the ingredients of Eq. 16 and subsequently turn to $T_{\|}$itself.

In order to compute reliable values for the kernel $U_{\|}$, one can make use of the static limit of Eq. 7, from which

$$
U_{\|}=\rho_{\mathrm{F}}^{-1}-\chi_{\|}^{-1}(0)
$$




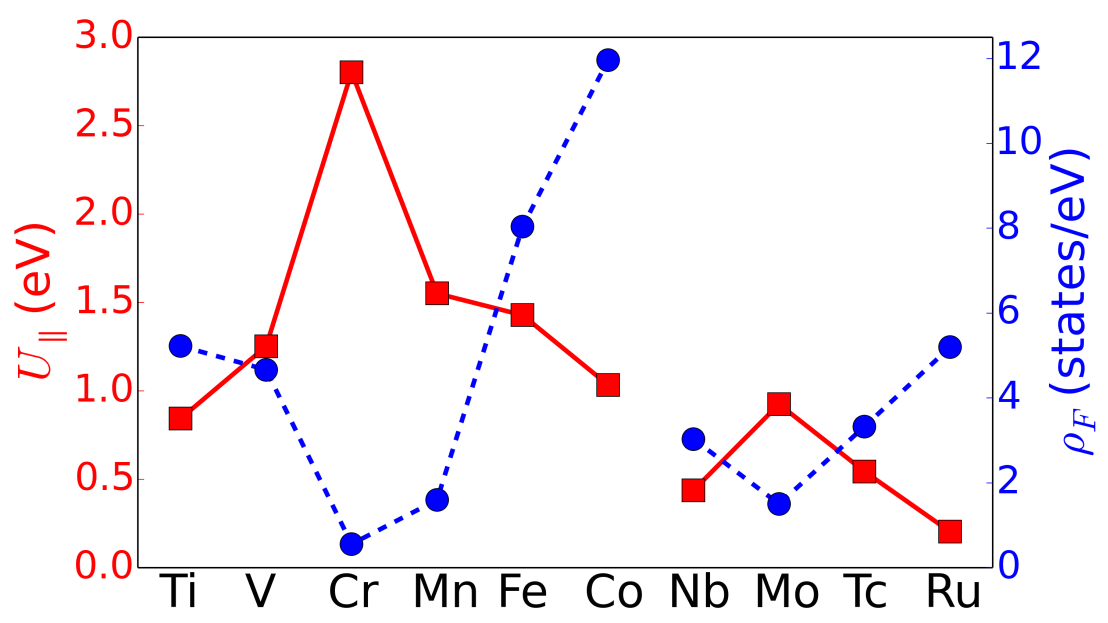

Figure 6: Calculated longitudinal kernel and DOS at $E_{F}$ for $3 d$ and $4 d$ magnetic adatoms deposited on $\operatorname{Ag}(100)$. Solid (red) and dashed (blue) lines show the values for $U_{\|}$(Eq. 17) and $\rho_{\mathrm{F}}$ whose corresponding ordinate axes are placed on the left (red) and right (blue) of the graph, respectively. Note that lines are broken in order to separate $3 d$ from $4 d$ elements.

We note that $\chi_{\|}(0)$ can be calculated by a standard ground state DFT calculation with a static magnetic field $\Delta B$ via $\chi_{\|}(0)=\Delta m / \Delta B$, with $\Delta m$ the corresponding self-consistent change of the magnetic moment [97]. In Fig. 6 we show the calculated values of $\rho_{\mathrm{F}}$ and $U_{\|}$for several $3 d$ and $4 d$ adatoms deposited on $\operatorname{Ag}(100)$. The most important message conveyed by this figure is the large variation of $U_{\|}$among different elements; while $U_{\|} \lesssim 0.5 \mathrm{eV}$ for most $4 d$ elements, $U_{\|} \gtrsim 1.5 \mathrm{eV}$ for various $3 d$ elements, reaching a maximum of one order of magnitude difference between Ru and Cr. A second important feature revealed by Fig. 6 is the distribution of $U_{\|}$ within each $d$-shell, whereby it is smallest at the ends of the row - case of Ti and Co among $3 d$, $\mathrm{Nb}$ and Ru among $4 d$ - and highest in the middle of the row - case of Cr and Mn among $3 d$, Mo and Tc among $4 d-$, yielding an approximate inverted V-shape. We note that $\rho_{\mathrm{F}}$ in Fig. 6 shows the opposite behavior, i.e. it is minimum for $\mathrm{Cr}$ and maximum for $\mathrm{Co}$ and $\mathrm{Ru}$. This is consistent with Eq. 17, although we note strong deviations from the $U_{\|} \propto \rho_{\mathrm{F}}^{-1}$ relationship (see in particular the case of $\mathrm{Cr}$ ), revealing the importance of the term $\chi_{\|}^{-1}(0)$ in Eq. 17.

The inverse of the static spin-susceptibility $\chi_{\|}^{-1}(0)$ is closely connected to the magnetic equation of state, i.e. the dependence of the energy $E$ as a function of the magnetic moment $m$, via [97],

$$
\chi_{\|}^{-1}(0)=\left.\frac{\partial^{2} E(m)}{\partial m^{2}}\right|_{m=m_{0}}<0 .
$$

In essence, the magnetic equation of state informs about how stable the magnetic solution is in comparison to the non-magnetic one. Fig. 7 shows the calculated $E(m)$ for the set of adatoms considered in Fig. 6 (calculations were carried out by employing DFT for a series of external magnetic fields [97]). This figure reveals that the energy difference between the non-magnetic and magnetic state,

$$
\Delta E=E(m=0)-E\left(m=m_{0}\right),
$$

is of the order of $\mathrm{eV}$ and can largely vary for different adatoms. Importantly, the calculations show that $3 d$ adatoms overall have a substantially larger $\Delta E$ than $4 d$ adatoms. For Cr, for 

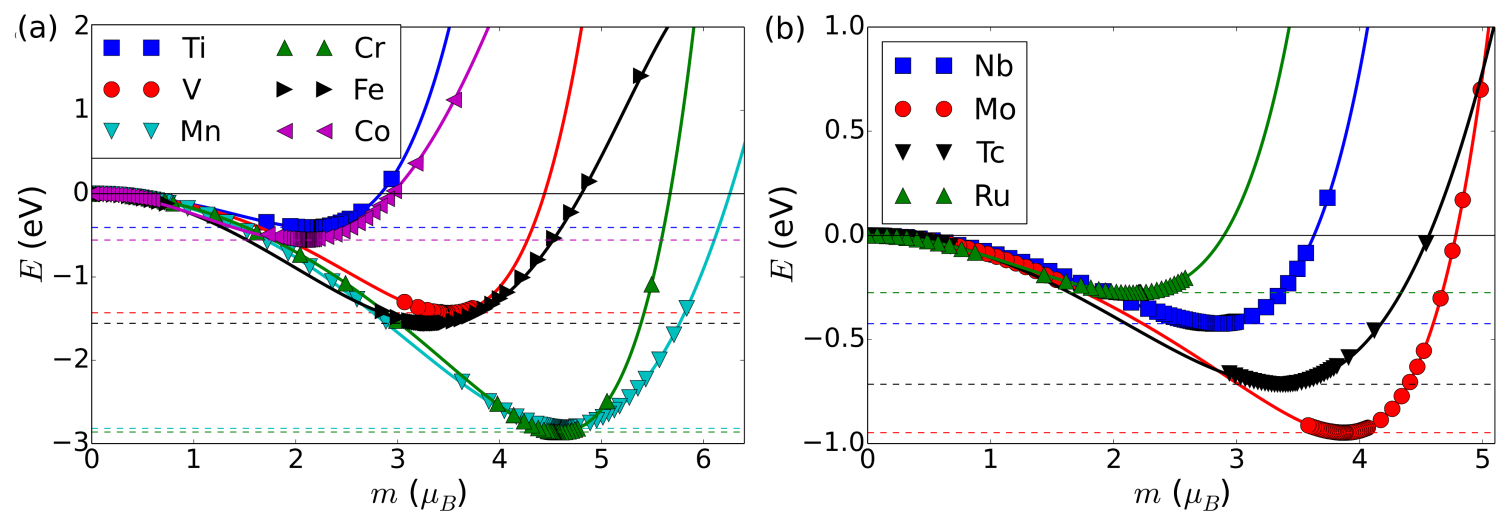

Figure 7: Calculated energy as a function of the adatom's magnetic moment. (a) and (b) $3 d$ and $4 d$ elements, respectively. DFT calculations are denoted by markers, while the solid lines are a fit to the calculations using $E=\sum_{i=1}^{4} a_{i} m^{2 i}$. The horizontal dashed lines mark the minimum energies for different adatoms.

instance, $\Delta E \sim 3 \mathrm{eV}$, while for $\mathrm{Ru} \Delta E \sim 0.25 \mathrm{eV}$. Therefore, DFT predicts most $3 d$ adatoms to be magnetically more stable than $4 d$ ones, as expected. Furthermore, given that Eq. 18 together with Eq. 17 relates the XC kernel to the second derivative of the equation of state at $M_{0}$, one can establish an approximate connection between the depth of the minimum of $E(M)$ and the value of $U_{\|}$, as it is visible from the comparison of Figs. 7 and 6; the deeper the minimum, the larger $U_{\|}$.

We note that following the above procedure, one can also extract the kernel in the non-magnetic ground state, i.e. the so-called Stoner XC parameter $I_{\mathrm{xc}}$ [98]. This can be achieved by considering the curvature of $E(M)$ not at $m=m_{0}$ but at $m=0$, as well as using the non-magnetic DOS in Eq. 17 instead of the magnetic one. As it is clearly visible from Fig. 7, the curvature is very different at $m=0$ and $m=m_{0}$. Furthermore, $\rho_{\mathrm{F}}$ can also strongly vary from a magnetic to a non-magnetic calculation. As a consequence, the distribution of $I_{\mathrm{xc}}$ along the transition metal series first reported by Janak in Ref. [98] is very different to that of $U_{\|}$illustrated in Fig. 6 .

Having analyzed the properties of $\rho_{\mathrm{F}}$ and $U_{\|}$, we next focus on the longitudinal relaxation time $T_{\|}$. The values calculated from Eq. 16 are plotted in Fig. 8(a) for $3 d$ and $4 d$ adatoms deposited on $\operatorname{Ag}(100)$ and $\mathrm{Cu}(111) . T_{\|}$is of the order of a few fs in all cases, being overall slightly larger for $4 d$ than $3 d$ adatoms, while the choice of substrate does not substantially affect it. Within each $d$-shell, $T_{\|}$is largest at the ends of the row while it is minimum for the half filled elements, thus resembling the behavior of $\rho_{\mathrm{F}}$ (compare Figs. 6 and 8(a)). Ru on $\mathrm{Ag}(100)$ has the highest value of $T_{\|} \sim 50 \mathrm{fs}$, mainly as a consequence of the denominator of Eq. 16 being closer to zero than in other elements. In contrast, $\mathrm{Cr}$ and Mn have $T_{\|} \sim 1 \mathrm{fs}$ in both substrates, i.e. nearly two orders of magnitude less than the aforementioned example. As a general feature, we note that the order of magnitude of $T_{\|}$is settled by the energy scale of the problem: all quantities involved in Eq. 16 are of the order of eV, whose corresponding time scale is fs. Therefore, the longitudinal relaxation of the spin considered here is extremely fast. The physical reason is the large exchange splitting dominating the relaxation process, which makes it energetically very expensive to modify the length of the moment due to the high energies involved. 

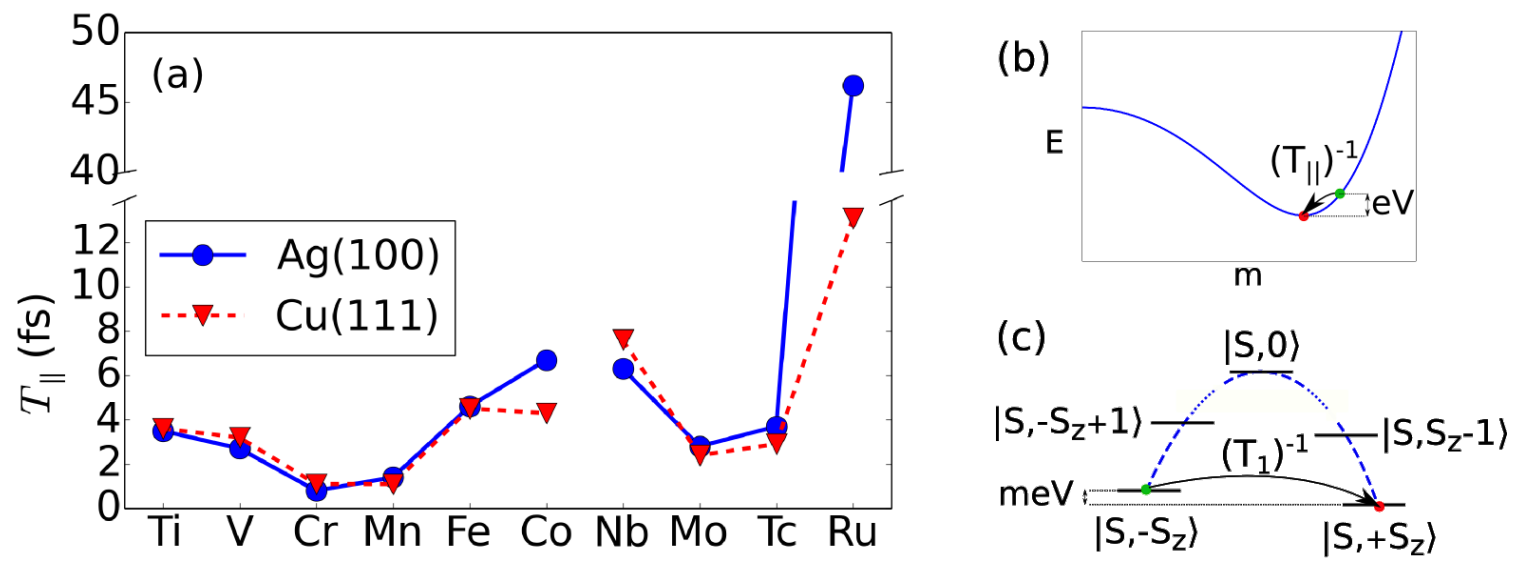

Figure 8: Longitudinal relaxation time. (a) Calculated values for the longitudinal relaxation time $T_{\|}$for magnetic $3 d$ and $4 d$ adatoms on $\mathrm{Ag}(100)$ (blue circles) and $\mathrm{Cu}(111)$ (red triangles). (b) and (c) Schematic illustration of two different processes contributing to the spin-relaxation time. Green and red dots respectively represent the excited and ground states. (b) Schematic description of the excitations contributing to $T_{\|}$considered in this work. These take place in a continuum energy landscape of order $\mathrm{eV}$ and are driven by direct spin-conserving electron-hole transitions. (c) Illustration of the prototypical energy diagram used to describe the experiments of Refs. [41, 57, 22, 72]. The initially degenerate two ground states of maximum $S_{z}$ become nondegenerate by energy $\sim \mathrm{meV}$ under externally applied magnetic fields of 1-10 T. Transitions between these two states then determine $T_{1}$, which take place via quantum tunneling.

\subsubsection{Connection to experimental measurements}

Let us next consider the experimental scenario regarding the measurement of the longitudinal spin-relaxation time. For this, we first note that in a experiment, several different mechanisms can contribute to this relaxation process, whose overall relaxation time is generally denoted as $T_{1}$. In this context, $T_{\|}$calculated here is a particular contribution to $T_{1}$, which may include further contributions depending on the physical processes taking place. To the best of our knowledge, the first experimental technique that measures $T_{1}$ in magnetic single adatoms was developed by Loth and co-workers [41]. Within this STM-based technique, the spin-relaxation time was measured by monitoring the decay of electrons in excited states after the application of an all-electronic pump-probe scheme [41]. It is noteworthy that this scheme has so far only been applied to adatoms deposited on semi-insulating substrates, which are close to the atomic limit. The original work by Loth and co-workers measured $T_{1} \sim 90 \mathrm{~ns}$ for a Fe-Cu dimer on $\mathrm{Cu}_{2} \mathrm{Ni} / \mathrm{Cu}(100)$ [41]. A subsequent work by Rau and co-workers measured $T_{1} \sim 200 \mu$ s for a single Co atom on $\mathrm{MgO} / \mathrm{Ag}(100)$ [57]. Lastly, Baumann and co-workers reported $T_{1} \sim 90 \mu \mathrm{s}$ for a single Fe atom on $\mathrm{MgO} / \mathrm{Ag}(100)$ [22], while in a recent work of Paul and co-workers on the same system, [72] the value of $T_{1}$ was enhanced up to the ms regime by fine-tuning external conditions such as the height of the STM tip. To conclude, we note that the reported time resolution of the measuring technique employed in the above experiments ranges between few ns to hundreds of ps.

All the above measured values of the spin-relaxation time are several orders of magnitude larger than the values of order fs that we discussed in this review for $T_{\|}$(see Fig. 8(a)). Let us first 
point out that all of the above experiments were performed under externally applied static magnetic fields that range between $1 \mathrm{~T}$ and $10 \mathrm{~T}$. This, in turn, breaks the degeneracy of the spin ground states [41], a situation that is commonly modeled by a shifted discrete energy diagram as the one shown in Fig. 8(c). We note that excitations within such a diagram are not allowed to change the length of the spin moment (spin quantum number $S$ in this context), but only its projection (magnetic quantum number $S_{z}$ ). Therefore, the main spin-relaxation process contributing to $T_{1}$ within such an scheme involves transitions between the two nondegenerate states with same $S$ but opposite $S_{z}$ (see Fig. 8(c)). We note that their energy separation is of order meV, hence much smaller than the excitations of order $\mathrm{eV}$ involved in the change of the spin magnetic moment size considered for the calculation of $T_{\|}$, as schematically depicted in Fig. 8(b). Furthermore, given that direct transitions between the two non-degenerate states of Fig. 8(c) are virtually nonexistent, spin-relaxation in these conditions is driven by quantum tunneling processes, which are intrinsically much slower than the direct transitions considered here. These two considerations explain why the spin-relaxation time measured under the mentioned experimental conditions is several orders of magnitude larger than the values of $T_{\|}$analyzed in this review.

It is apparent that, in order to experimentally access the dynamics encoded into $T_{\|}$, a measuring scheme based on ultrafast techniques that modify the length of the spin magnetic moment is required. Considering the technological developments within STM measuring techniques [99, $100,101]$, accessing the fs time scale of magnetic adatoms seems to be a reasonable goal for the near future by, e.g. using ultrafast laser pulses, a breakthrough that would allow to monitor the ultrafast spin-dynamics analyzed here.

\subsection{Transverse component}

While longitudinal spin-susceptibility contains excitations that modify the spin density, the transverse one describes damped precessional motion of the spin moment [96]. This motion, in turn, is described by the imaginary part of the enhanced spin-susceptibility, $\operatorname{Im} \chi_{ \pm}(\omega)$, a quantity that has been analyzed in depth in Sec. 3 (in particular, see Fig. 2). The characteristic form of the spin-excitation hosted by $\operatorname{Im} \chi_{ \pm}(\omega)$ and its connection to the transverse spin-relaxation time is illustrated in Fig. 9(a). As discussed in Sec. 3, $\operatorname{Im} \chi_{ \pm}(\omega)$ is mainly determined by the resonance frequency, $\omega_{\text {res }}$, which is settled by the SOC, and the width of the spin-excitation, $\Gamma$, which is proportional to the damping term $\eta$ (see in particular Eq. 52 in Appendix B). Noteworthily, the main contribution of the hybridization to $\Gamma$ is proportional to the electron-hole excitations of opposite spin channel, $n_{\mathrm{e}-\mathrm{h}}^{\prime}=\pi \rho_{\mathrm{F}, \uparrow} \rho_{\mathrm{F}, \downarrow}[80]$ :

$$
\Gamma \simeq \frac{n_{\mathrm{e}-\mathrm{h}}^{\prime}}{Q} \omega_{\mathrm{res}}
$$

with $Q=\operatorname{Re} \partial \chi_{ \pm}^{\mathrm{KS}}(\omega) /\left.\partial \omega\right|_{\omega=0}$. We note that the order of magnitude of $\omega_{\text {res }}$ ranges between $10^{-2}-1 \mathrm{meV}$ while $n_{\mathrm{e}-\mathrm{h}}^{\prime} / Q$ is a unitless fraction that is typically of order unity.

Importantly, a finite width corresponds to a finite damping of the precessing magnetic moment and is thus directly linked to the transverse spin-relaxation time (see Appendix B and Eq. 20):

$$
T_{\perp}=\frac{2}{\Gamma} \propto\left(n_{\mathrm{e}-\mathrm{h}}^{\prime}\right)^{-1} .
$$



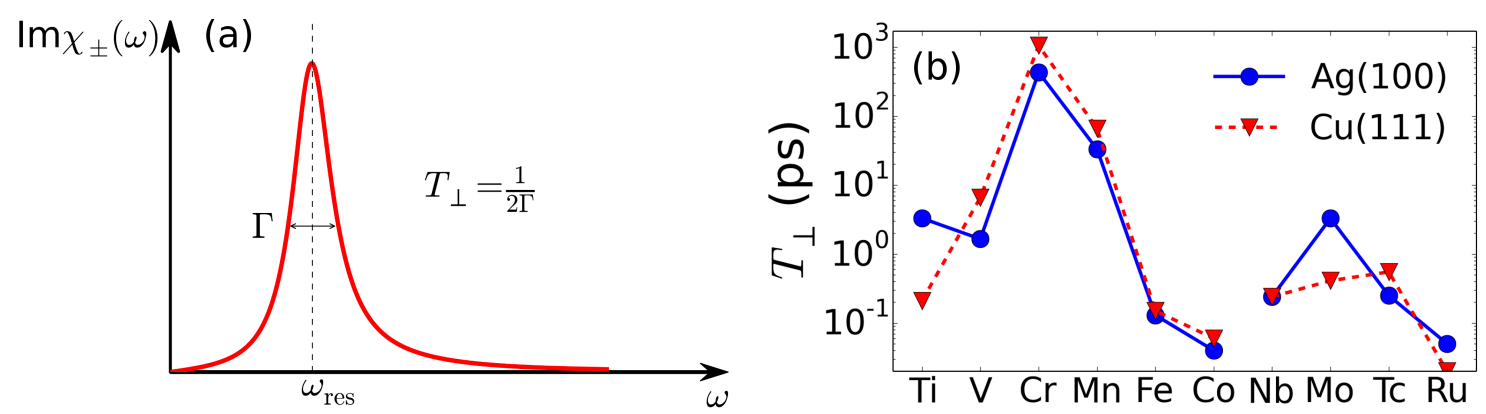

Figure 9: Transverse relaxation time. (a) Schematic illustration of a transverse spin-excitation of a single adatom contained in $\operatorname{Im} \chi_{ \pm}(\omega)$. The resonance frequency, $\omega_{\text {res }}$, and the width, $\Gamma$, are indicated in the figure. (b) Calculated values for the transverse relaxation time $T_{\perp}$ for magnetic $3 d$ and $4 d$ adatoms on $\mathrm{Ag}(100)$ (blue circles) and $\mathrm{Cu}(111)$ (red triangles). Note the logarithmic scale in the y axis.

We note that, while $T_{\|}$in Eq. 16 is directly proportional to the density of spin-conserving electron-hole excitations $n_{\mathrm{e}-\mathrm{h}}, T_{\perp}$ above is inversely proportional to the spin-flip counterpart $n_{\mathrm{e}-\mathrm{h}}^{\prime}$. Let us also note that an expression with similar physical implications has been derived in the context of spin models with Kondo exchange in open quantum systems, the so-called non-adiabatic contribution to the spin-decoherence rate [23] (see in particular Eq. (70) in that reference).

In Fig. 9(b) we show calculated values for $T_{\perp}$ extracted from the corresponding spin-excitation width (see Fig. 2). We note that the variation of $T_{\perp}$ among adatoms is considerably larger (up to two orders more) than that of $T_{\|}$, shown in Fig. 8(a). This is a consequence of the large variation of the width $\Gamma$ of atomic spin-excitations, which can range from $10^{-2} \mathrm{meV}$ to few meV $[53,81]$, i.e. nearly three orders of magnitude change. This, in turn, can be linked to the electronic DOS at the Fermi level via Eq. 20; adatoms where the DOS peak of the $d$-states lies close to the Fermi level, case of Ti, V, Fe and Co in Fig. 1(c), tend to be much more hybridized than those where only the tail of the DOS peak lies at the Fermi level, case of $\mathrm{Cr}$ and $\mathrm{Mn}$ in Fig. 1(c). In this way, $\mathrm{Cr}$ and $\mathrm{Mn}$ acquire large relaxation times of $T_{\perp} \sim 10^{1}-10^{2} \mathrm{ps}$, while strongly hybridized adatoms such as $\mathrm{Co}, \mathrm{Nb}$ and $\mathrm{Ru}$ have $T_{\perp} \sim 10^{-1}-10^{-2}$ ps. In all cases, the transverse spin-relaxation process is orders of magnitude slower than the longitudinal one, $T_{\perp} \gg T_{\|}$.

To conclude, let us note that the trend of $T_{\perp}$ within each $d$-shell row is opposite to that shown by $T_{\|}$(see Fig. 8(a)). This comes as a consequence of the dependence on the density of electronhole excitations, with $T_{\|} \propto n_{\mathrm{e}-\mathrm{h}}$ (see Eq. 16) and $T_{\perp} \propto\left(n_{\mathrm{e}-\mathrm{h}}^{\prime}\right)^{-1}$ (see Eq. 20). In fact, $T_{\|}$and $T_{\perp}$ can be formally related to each other considering the relation between the spin-conserving and spin-flip electron-hole contributions, namely $\rho_{\mathrm{F}}^{2} / 2=n_{\mathrm{e}-\mathrm{h}}+n_{\mathrm{e}-\mathrm{h}}^{\prime}$. From Eq. 16 for $T_{\|}$and Eqs. 20 and 21 for $T_{\perp}$ one can then infer the following expression:

$$
\frac{\rho_{\mathrm{F}}^{2}}{2} \simeq T_{\|} \frac{U_{\|} \rho_{\mathrm{F}}-1}{U_{\|}}+\frac{2}{T_{\perp}} \frac{Q}{\omega_{\text {res }}} .
$$

The unifying concept behind the above relationship between $T_{\|}$and $T_{\perp}$ is the hybridization of substrate electrons with the $d$-states of the transition metal adatoms, which in essence gives rise 
to a finite total $\rho_{\mathrm{F}}$. However, despite the formal relationship, the fact that $T_{\|}$and $T_{\perp}$ in Eq. 22 have fundamentally different prefactors makes the time scale of the two relaxation constants differ by nearly three orders of magnitude.

\subsubsection{Connection to experimental measurements}

Next, we consider several experimental measurements of spin-excitation lifetimes of different single adatoms and connect them to this work. The lifetime of an atomic spin-excitation can be experimentally accessed from the width of the step observed in IETS $\mathrm{d} I / \mathrm{d} V$ measurements, which provides a measure of $\Gamma$. Given that the energy resolution of this technique is $10^{-1} \mathrm{meV}$ at best [9], the longest lifetimes that can be inferred following this procedure are of order 10 ps (see Eq. 21). This type of experiments can measure adatoms deposited on both metallic and semi-insulating substrates; as a general trend, the latter induce a larger lifetime than the former due to a far smaller electronic hybridization. We begin by considering Ref. [8], where Khajetoorians and co-workers estimate the spin-excitation lifetime of a Fe adatom deposited on metallic $\mathrm{Cu}(111)$ to be $0.2 \mathrm{ps}$, in very good quantitative agreement with the calculated value $T_{\perp}=0.15 \mathrm{ps}$ for the same system (see Fig. 9(b)). Noteworthily, when the same atom is deposited on metallic $\mathrm{Pt}(111)$, the measured lifetime is increased by more than an order of magnitude $[9,14]$. Note that one finds a similar variation between the two substrates considered here for the elements Ti, V, Cr and Mo, as it can be checked in Fig. 9(b). Focusing next on the semi-insulating $\mathrm{Cu}_{2} \mathrm{Ni} / \mathrm{Cu}(100)$ substrate, a lower bound of $\sim 10$ ps has been experimentally estimated for Fe [3, 71], Mn [71] and Co [40] adatoms, although it is possible that the actual lifetimes are substantially larger. In fact, the calculations on $\mathrm{Cr}$ and $\mathrm{Mn}$, which are the elements with smallest hybridization and thus the ones closest to the semi-insulating limit, show that $T_{\perp}$ can reach up to $10^{3}$ ps (see Fig. 9(b)); hence, it is not unlikely that the lifetimes of the aforementioned adatoms on $\mathrm{Cu}_{2} \mathrm{Ni} / \mathrm{Cu}(100)$ could be of the same order of magnitude. Last, it is worth noting the case of $\mathrm{Co}$ on $\mathrm{MgO}$ [57], which, despite being a semi-insulating substrate, yields a relatively short spin-excitation lifetime of $\sim 0.5 \mathrm{ps}$, i.e. a common value for adatoms deposited in metallic substrates analyzed in this review (see Fig. 9(b)).

\section{$5 \quad$ Paramagnetic spin-excitations}

In this last section, we switch the main subject of study from magnetic to nonmagnetic adatoms, i.e., adatoms where the Stoner criterion for magnetism is nearly fulfilled. In particular, we analyze their dynamical properties, which can exhibit fingerprints of magnetism in the form of well-defined features in the spin-excitation spectrum, i.e., paramagnetic spin-excitations (PSE). Noteworthily, these excitations can be viewed as persistent spin-fluctuation modes that are activated by temperature and thus produce a measurable impact on properties such as specific heat or electron effective-mass enhancement [73, 67]. Thus, the same concept that leads to the destabilization of the magnetic moment seen in Sec. 3 can in this case instead be used to generate a signal of magnetic origin.

As in previous sections, the central quantity for the discussion is the spin-excitation spectrum, 

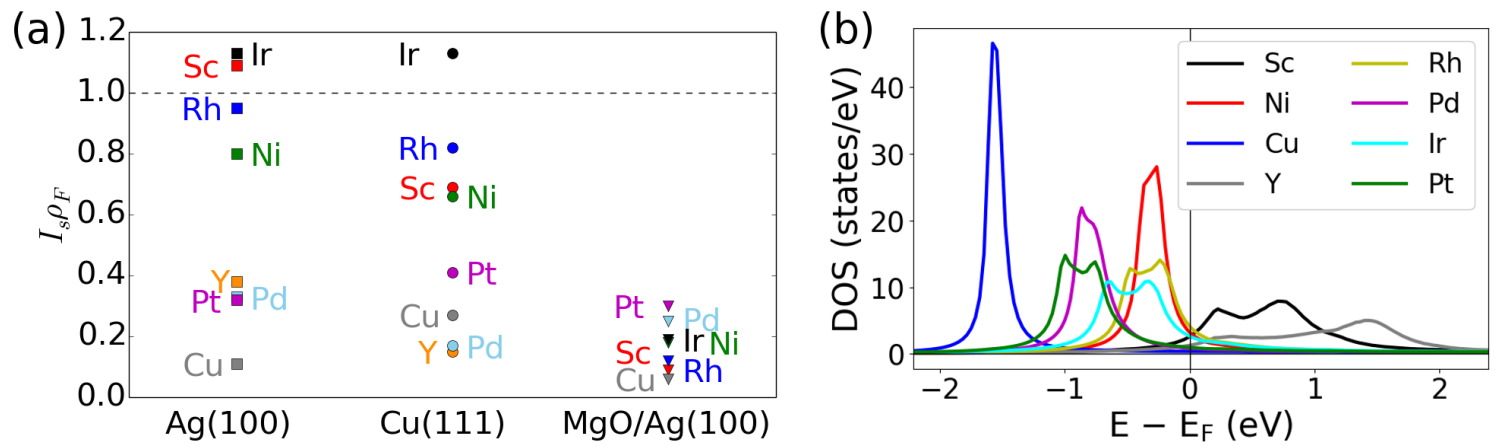

Figure 10: Ground state electronic properties of various $3 d, 4 d$ and $5 d$ transition metal adatoms. (a) Calculated Stoner product for adatoms deposited on $\mathrm{Ag}(100)$ (squares), $\mathrm{Cu}(111)$ (circles) and $\mathrm{MgO} / \mathrm{Ag}(100)$ (triangles). (b) Calculated non-magnetic DOS of adatoms on $\mathrm{Ag}(100)$. The vertical line denotes the Fermi level.

which in this case is encoded into the paramagnetic spin-susceptibility (see Eq. 7): [69]

$$
\chi(\omega)=\frac{\chi^{\mathrm{KS}}(\omega)}{1-I_{\mathrm{s}} \chi^{\mathrm{KS}}(\omega)} .
$$

Above, $I_{\mathrm{S}}$ denotes the so-called Stoner parameter, which plays the role of the exchange-correlation kernel in the adiabatic local spin-density approximation. It is noteworthy that the static limit of Eq. 23 recovers a local form of the familiar Stoner theory that provides the well-known criterion for magnetism, i.e.

$$
\left.\frac{\partial^{2} E(m)}{\partial m^{2}}\right|_{m=0}=\chi(0)^{-1}<0 \Rightarrow I_{\mathrm{S}} \rho_{\mathrm{F}}>1,
$$

where we used $\chi^{\mathrm{KS}}(0)=\rho_{\mathrm{F}}$ [69]. In essence, the product $I_{\mathrm{S}} \rho_{\mathrm{F}}$ quantifies the competition between the exchange interaction, which enhances the tendency towards magnetism of electrons in localized orbitals, and substrate hybridization, which induces delocalization of the adatom's electrons and therefore acts against magnetism, thus playing the role of the kinetic energy in the standard Stoner theory. It is interesting to note that even if an adatom does not fulfill the Stoner criterion, it can still develop dynamical PSE provided the details of the electronic structure make the denominator of Eq. 23 vanishingly small at a finite frequency.

\subsection{Searching for the optimal candidates via the Stoner criterion}

We begin by characterizing the set of $3 d, 4 d$ and $5 d$ transition metal adatoms that could potentially exhibit PSE. For this purpose, we consider the Stoner criterion of Eq. 24 and search for adatoms whose Stoner product is close to 1 . In Fig. 10(a) we plot $I_{\mathrm{S}} \rho_{\mathrm{F}}$ for several adatoms deposited on the $\mathrm{Ag}(100)$ and $\mathrm{Cu}(111)$ substrates analyzed throughout the review. In addition, we have also included the $\mathrm{MgO} / \mathrm{Ag}(100)$ substrate in order to analyze the differences introduces by a semi-insulating layer. We first note that the Stoner products of all the adatoms are below or slightly above 1 . As a general trend, the calculations show that the metallic substrates $\mathrm{Ag}(100)$ and $\mathrm{Cu}(111)$ host adatoms whose Stoner product is closer to the critical value 1 as compared to semi-insulating $\mathrm{MgO} / \mathrm{Ag}(100)$. This is mainly due to the small $\rho_{\mathrm{F}}$ in the latter, as tabulated in Table 2. Among the two metallic substrates, $\mathrm{Ag}(100)$ hosts adatoms whose Stoner product 


\begin{tabular}{|c|c|c|c|c|c|c|c|c|}
\hline & $\mathrm{Sc}$ & $\mathrm{Ni}$ & $\mathrm{Cu}$ & $\mathrm{Y}$ & $\mathrm{Rh}$ & $\mathrm{Pd}$ & $\mathrm{Ir}$ & $\mathrm{Pt}$ \\
\hline$\rho_{\mathrm{F}}($ states $/ \mathrm{eV})$ & 2.65 & 3.04 & 0.35 & 1.37 & 3.51 & 0.77 & 2.81 & 0.89 \\
\hline$I_{\mathrm{S}}(\mathrm{eV})$ & 0.41 & 0.29 & 0.32 & 0.28 & 0.27 & 0.39 & 0.40 & 0.36 \\
\hline$I_{\mathrm{S}} \rho_{\mathrm{F}}$ & 1.09 & 0.88 & 0.11 & 0.38 & 0.95 & 0.33 & 1.12 & 0.32 \\
\hline
\end{tabular}

Table 2: Calculated values for the DOS at the Fermi level $\left(\rho_{\mathrm{F}}\right)$, Stoner parameter $\left(I_{\mathrm{S}}\right)$ and Stoner product $\left(I_{\mathrm{S}} \rho_{\mathrm{F}}\right)$ for several $3 d, 4 d$ and $5 d$ adatoms on $\mathrm{Ag}(100)$.

(a)

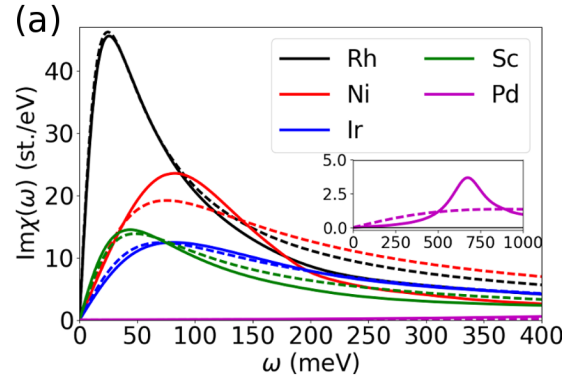

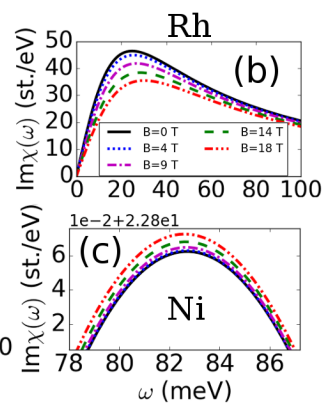

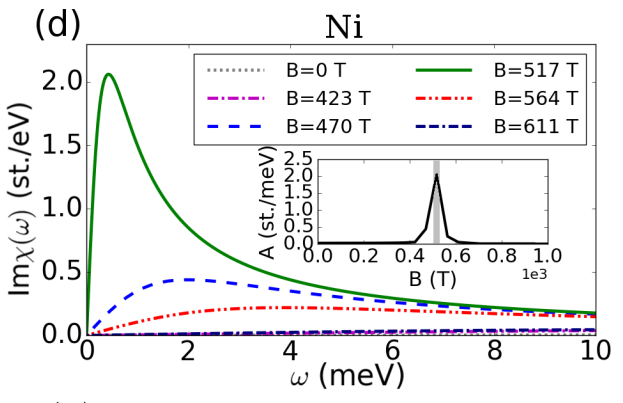

Figure 11: Spin-excitation spectrum of selected adatoms. (a) Solid lines illustrate the calculated density of PSE as given by $\operatorname{Im} \chi(\omega)$ (Eq. 23) of selected $3 d, 4 d$ and $5 d$ transition metal adatoms deposited on the metallic $\operatorname{Ag}(100)$ substrate. Dashed lines denote the approximation of Eq. 25. Note that both Eqs. 23 and 25 give rise to PSE. (b) and (c) Density of PSE as given by $\operatorname{Im} \chi(\omega)$ for $\mathrm{Rh}$ and $\mathrm{Ni}$ adatoms, respectively, for magnetic fields of up to $18 \mathrm{~T}$ (both figures share the same legend). (d) Same as in (c) but for larger magnetic fields of up to $10^{3} \mathrm{~T}$. The inset depicts the evolution of the PSE's amplitude (see Eq. 26) as a function of the magnetic field.

are closest to 1 , with $I_{\mathrm{S}} \rho_{\mathrm{F}}$ ranging between $\sim[1-0.1,1+0.1]$ for $\mathrm{Sc}, \mathrm{Ir}, \mathrm{Rh}$ and Ni adatoms. Therefore, in the rest of this Section we focus on discussing the $\operatorname{Ag}(100)$ substrate in detail, as it illustrates best the concept of PSE.

In Fig. 10(b) we show the calculated DOS of the considered $3 d, 4 d$ and $5 d$ adatoms. The figure shows that the large peaks coming from localized $d$-states acquire a finite width, which ranges from $\sim 0.2 \mathrm{eV}$ in the case of $\mathrm{Cu}$, up to $\sim 1 \mathrm{eV}$ in the case of $\mathrm{Sc}$ and $\mathrm{Y}$. This feature reveals a strong hybridization of the adatom's $d$-states with the electron bath of the substrate $[52,80]$. As a consequence, the DOS acquires a finite value at the Fermi level, $\rho_{\mathrm{F}}$, which is listed in Table 2 for all adatoms, alongside with the calculated Stoner parameter and the resulting Stoner product. We note that the largest $\rho_{\mathrm{F}}$ corresponds to Rh due to the large width of the nearly filled $d$-peak in the DOS and its proximity to the Fermi level (see Fig. 10(b)). It is also noteworthy that Sc and Ir, which are predicted to be slightly magnetic, have a smaller $\rho_{\mathrm{F}}$ than Rh and Ni. This is compensated by the Stoner parameter, which is almost $50 \%$ larger as compared to $\mathrm{Ni}$ and $\mathrm{Rh}$. In this respect, the case of $\mathrm{Ni}$ is specially interesting, given that the calculated $I_{\mathrm{S}}$ for the adatom is almost $50 \%$ smaller compared to the bulk value [98]. This drop of $I_{\mathrm{S}}$ is the reason why $\mathrm{Ni}$ is magnetic in bulk but non-magnetic as an adatom on $\operatorname{Ag}(100)$ and other metallic substrates [102, 103]. 


\subsection{Fingerprints of magnetism in the dynamical regime}

In Fig. 11(a) we illustrate the calculated spin-excitation spectra as given by $\operatorname{Im} \chi(\omega)$ from Eq. 23, where all calculations were done considering the non-magnetic ground state. Interestingly, Fig. 11(a) reveals peak-like structures resonating at frequencies below $100 \mathrm{meV}$ for $\mathrm{Rh}, \mathrm{Ni}$, Ir and Sc adatoms. This is exceptional, as most non-magnetic elements exhibit a featureless spectrum owing to a complete overdamping of the excitations. Rh represents the most favorable case, displaying a well-defined peak at $\omega_{\text {res }} \sim 20 \mathrm{meV}$ and a width of $\Delta \sim 50 \mathrm{meV}$, the associated lifetime being $\sim 30 \mathrm{fs}$. It is noteworthy that both the lifetime and the height of the peak, the latter being related to the intensity of the excitation, are only one order of magnitude smaller than those of usual transverse spin-excitations of magnetic adatoms studied in Sec. 3 (see in particular Fig. 2) and that are accessible by IETS (see, e.g., Fe on $\mathrm{Cu}(111)$ in Refs. $[8,53]$ ). On the other extreme, Pd in Fig. 11(a) shows a highly overdamped resonance at around $600 \mathrm{meV}$ (see figure inset) whose intensity is an order of magnitude smaller than that of Rh. Therefore, the calculations reveal the existence of PSE whose resonance frequency and width vary strongly depending on the adatom.

Next, we focus on characterizing the physical mechanism behind PSE that allows an interpretation of the $a b$ initio results displayed in Fig. 11(a). For this purpose, let us consider the frequency expansion of the paramagnetic KS spin response function up to linear order, i.e., $\chi^{\mathrm{KS}}(\omega)=\rho_{\mathrm{F}}+\mathrm{i} \alpha \omega+\mathcal{O}\left(\omega^{2}\right)$. One can show [77] that the linear expansion coefficient is well approximated by $\alpha \sim-\pi \rho_{\mathrm{F}}^{2} / 4$. Therefore, the spin-excitation spectrum within this approximation is given by a simple expression involving only the DOS at $E_{\mathrm{F}}$ and the Stoner parameter:

$$
\operatorname{Im} \chi(\omega)=\frac{\pi}{4} \frac{\rho_{\mathrm{F}}^{2} \omega}{\left(1-I_{\mathrm{S}} \rho_{\mathrm{F}}\right)^{2}+\left(\frac{\pi}{4} I_{\mathrm{S}} \rho_{\mathrm{F}}^{2} \omega\right)^{2}} .
$$

The above expression can be readily computed by using the calculated values for $\rho_{\mathrm{F}}$ and $I_{\mathrm{S}}$ shown in Table 2: the results are displayed in Fig. 11(a) (see dashed lines). A comparison to the full $a b$ initio calculations (solid lines) reveals a very good agreement for frequencies below $100 \mathrm{meV}$ in the case of $\mathrm{Rh}$, Ir and $\mathrm{Sc}$, where both the peak and width are properly described within $\leqslant 10 \%$ relative error. This error is considerably larger in the case of $\mathrm{Ni}$, indicating the importance of higher order expansion terms in $\omega$ for this case. Finally, the peak for Pd is far beyond the limit of small frequencies and therefore the approximation of Eq. 25 breaks down.

Proving Eq. 25 to be an accurate approximation of the full spin-excitation density given by Eq. 23 is extremely convenient, as the former provides an analytical interpretation for the origin of PSE in terms of just $\rho_{\mathrm{F}}$ and $I_{\mathrm{s}}$, two basic electronic properties of adatoms. Indeed, the resonance frequency, linewidth and amplitude of PSE predicted by Eq. 25 can be cast into simple expressions:

$$
\begin{gathered}
\omega_{\text {res }}=\frac{4}{\pi} \frac{\left|1-I_{\mathrm{s}} \rho_{\mathrm{F}}\right|}{I_{\mathrm{s}} \rho_{\mathrm{F}}^{2}}, \Delta=2 \sqrt{3} \omega_{\mathrm{res}}, \\
A \equiv \operatorname{Im} \chi\left(\omega_{\mathrm{res}}\right)=\frac{1}{2 I_{\mathrm{S}}\left|1-I_{\mathrm{s}} \rho_{\mathrm{F}}\right|} .
\end{gathered}
$$

Interestingly, a potential measurement of the above quantities would directly yield experimental estimates for $\rho_{\mathrm{F}}$ and $I_{\mathrm{S}}$. In closer inspection, one recognizes the Stoner product $I_{\mathrm{S}} \rho_{\mathrm{F}}$ as the key quantity in Eq. 26; as $I_{\mathrm{s}} \rho_{\mathrm{F}} \rightarrow 1$ (i.e., magnetic instability), the resonance frequency as 


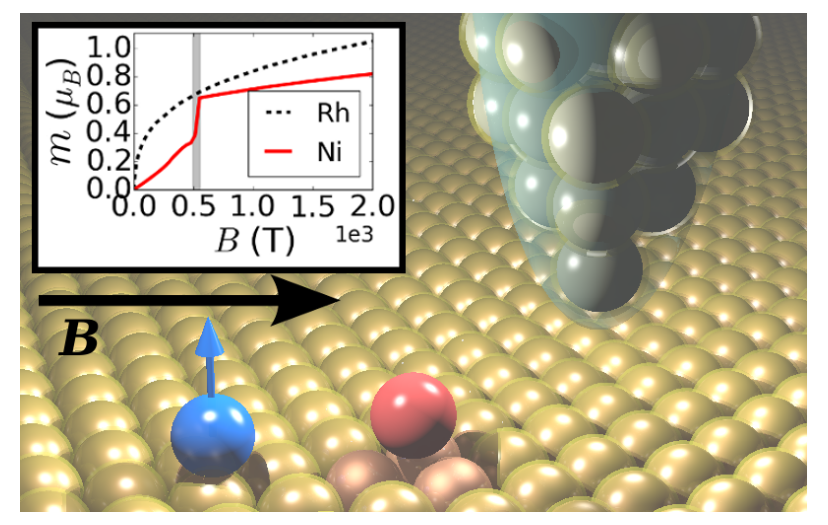

Figure 12: Minimal setup illustrating the proposed IETS measurement. Substrate, nonmagnetic adatom and tip atoms are displayed as gold, red and grey balls, respectively, while the black arrow depicts an external magnetic field $B$. The graph in the inset illustrates the calculated magnetic moment $m$ as a function of the external field for $\mathrm{Rh}$ and $\mathrm{Ni}$ adatoms, with the grey area indicating the critical regime of $\mathrm{Ni}$ where $m$ shows a discontinuity. A blue ball with an arrow has been added in the main figure to illustrate the possibility of coupling a magnetic adatom to the non-magnetic one, inducing on the latter a magnetic moment of the order of the values shown in the inset, thus mimicking the effect of large magnetic fields [104].

well as the linewidth tend to zero while the intensity of PSE shows a singularity. This analysis offers therefore the interpretation we seeked for, namely that elements closer to the magnetic instability show enhanced PSE, as it can be clearly checked from the comparison of Figs. 10(a) and 11(a). We emphasize that the mechanism just described is fundamentally different from the one taking place in the magnetic adatoms analyzed in the previous sections, where the resonance frequency of transverse spin-excitations is settled by the SOC via the MAE.

\subsection{Potential means of measurement: connection to $\mathrm{dI} / \mathrm{dV}$ curves}

We next focus on assessing the potential impact of PSE on the $\mathrm{d} I / \mathrm{d} V$ signal as measured in IETS experiments, the technique of choice for measuring magnetic excitations (see, e.g., Refs. $[8,9,105])$. The corresponding minimal setup is illustrated in Fig. 12, which displays an STM tip measuring the adatom's excitations under an applied external magnetic field, denoted as $B$. We first notice that PSE respond to magnetic fields by shifting their resonance frequency. This is quantitatively demonstrated in Figs. 11(b) and 11(c), where the calculated spin-excitation spectra are shown for $\mathrm{Rh}$ and $\mathrm{Ni}$ adatoms, respectively, under $B$ fields of $\sim 10 \mathrm{~T}$ that are achieveable in state-of-the-art laboratories (see, e.g., Refs. $[9,57,38]$ ). Noteworthily, while the PSE of Rh shifts towards larger frequencies as $B$ is increased (see Fig. 11(b)), the PSE of Ni exhibits the opposite behavior (see Fig. 11(c)). This difference arises from the fact that magnetic fields induce an effective modification of Stoner product, i.e., $I_{\mathrm{s}} \rho_{\mathrm{F}} \rightarrow \xi(B) I_{\mathrm{S}} \rho_{\mathrm{F}}$, where $\xi(B)$ is a term that depends both on the magnetic field as well as on the adatom's electronic structure [77]. In particular, the details of the latter make $\xi(B)>1$ for Ni while $\xi(B)<1$ for Rh [77], leading to the aforementioned divergent responses in accordance with Eq. 26.

Remarkably, when strong enough magnetic fields are applied to Ni, the modified Stoner criterion 

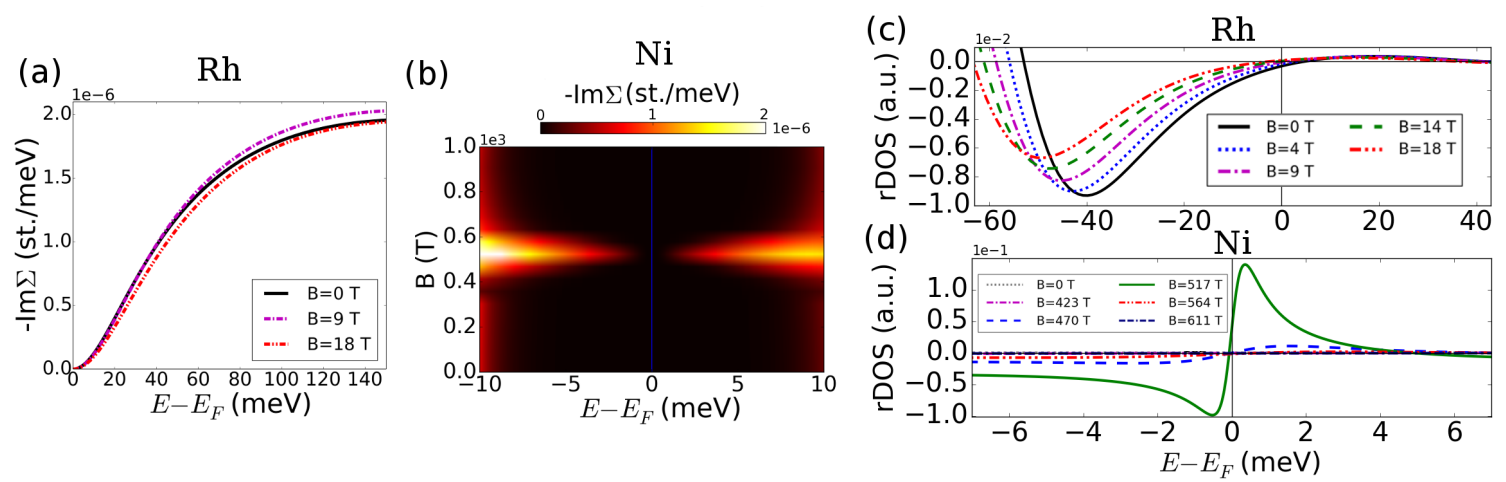

Figure 13: Calculated electronic renormalization. (a) and (b) Imaginary part of the self-energy, $\operatorname{Im} \Sigma\left(V_{\mathrm{F}}\right)$, for $\mathrm{Rh}$ and $\mathrm{Ni}$ adatoms, respectively. Note the different scale of the magnetic field and the energy window in the two cases. Vertical (blue) line in (b) separates negative and positive energies. (c) and (d) Energy derivative of the renormalized DOS (s orbital) for Rh and Ni, respectively. Note the difference in magnitude on the applied magnetic fields in both cases.

can be tuned towards the critical point, as shown in Fig. 11(d). As a consequence, the PSE's resonance frequency approaches the origin in a singular way while the amplitude of the excitation is enhanced by as much as two orders of magnitude for $B \sim 500 \mathrm{~T}$. It is interesting to note that this critical behavior is also present on the $B$-field dependence of the induced magnetic moment $m$, as shown in the inset of Fig. 12. While Rh shows a continuous dependence, Ni reveals a discontinous transition at approximately the critical field value $B \sim 500 \mathrm{~T}$, above which the system enters a magnetic regime where the internal exchange field effectively contributes to $m$ on top of the external Zeeman field, featuring the atomic version of a quantum phase transition. We note that, although such large $B$ fields are clearly out of reach for current experiments, this feature could be potentially observed, e.g., via the proximity effect, by placing a magnetic adatom in the neighborhood of the non-magnetic one (see Fig. 12). Calculations show that the former can induce on the latter a magnetic moment of the same order of magnitude as the one induced by the fields of Fig. 11(d) [104], thus mimicking the action of large magnetic fields.

Next we evaluate the impact of PSE on the $\mathrm{d} I / \mathrm{d} V$ signal of an IETS measurement. For such purpose we consider the so-called Tersoff-Hamann approximation [106, 107], which relates the tunneling conductance to the electronic DOS at the tip position, which is renormalized by the adatom's excitations. The latter quantity can be accessed by means of a technique that combines many-body perturbation theory with the TDDFT scheme; details can be found in Ref. [54]. The central object within this formalism is the electron self-energy, $\Sigma$, which contains the interactions between the tunneling electrons from the tip at bias voltage $V$ and the adatom's PSE. It is particularly revealing to inspect its imaginary part [54],

$$
\operatorname{Im} \Sigma\left(V_{\mathrm{F}}\right)=-I_{s}^{2} \int_{0}^{-V} \mathrm{~d} \omega \rho\left(V_{\mathrm{F}}+\omega\right) \operatorname{Im} \chi(\omega)
$$

with $\rho(E)$ the energy-dependent DOS, $V_{\mathrm{F}}=E_{\mathrm{F}}+V$ and $E_{\mathrm{F}}$ the Fermi energy. The calculated $\operatorname{Im} \Sigma\left(V_{\mathrm{F}}\right)$ is shown in Fig. 13(a) for Rh under various magnetic fields of up to $18 \mathrm{~T}$. The results reveal a clear step for positive bias voltage that saturates at $\sim 100 \mathrm{meV}$, i.e., after the PSE peak has been integrated (see Eq. 27). Note also that the calculated self-energy slightly varies as a function of the magnetic field. When larger magnetic fields are applied, as illustrated in 
Fig. 13(b) for the case of Ni, the critical behavior of the PSE (see Fig. 11(d)) translates into a clear maximum at the value of the critical field, where $\operatorname{Im} \Sigma\left(V_{\mathrm{F}}\right)$ increases by an order of magnitude.

The presence of PSE has a broad effect on the renormalization of the DOS at the vacuum, where IETS tips measure the signal. In particular, the energy derivative of the renormalized DOS (rDOS) is a quantity that is linked to the $\mathrm{d}^{2} I / \mathrm{d} V^{2}$ curve measured by IETS [54]. The former quantity is displayed in Fig. 13(c) for Rh, where the magnetic field dependence is clearly visible. Noteworthily, the calculations demonstrate that the tunneling electrons from the tip are able to trigger the PSE, leading to a peak in the meV region that, furthermore, reacts to external magnetic fields by shifting its resonance frequency as well as substantially modifying its intensity. We also note the strong asymmetric distribution between positive and negative frequencies, a feature that emerges from the background electronic structure [54] and is commonly present in $\mathrm{d}^{2} I / \mathrm{d} V^{2}$ curves measured on magnetic adatoms (see, e.g., Refs. $[1,8,108,9,109]$ ). On the other hand, when $\mathrm{Ni}$ is driven into the critical regime as in Fig. 13(d), the calculations reveal a huge change of the signal's intensity as the PSE approaches the critical point. This analysis therefore shows that magnetism offers a prime way of manipulating PSE, enabling to discern them from other excitations of similar energy but non-magnetic origin, such as phonons.

\section{Outlook}

In this review, we have presented a state-of-the-art treatment based on TDDFT of several dynamical aspects of single-adatoms deposited on metallic substrates. As the first main topic, we have shown that the magnetic stability defined by the MAE can be severely reduced as a consequence of quantum spin-fluctuations that arise mainly from Stoner spin-flip excitations. Our analysis has quantitatively shown how protection against fluctuations is gained by reducing the hybridization with substrate electrons as well as maximizing the adatom's local magnetic moment. In this context, future work should address an important contribution that has not been considered here, namely the vibrational contribution of phonons, which is believed to be key in systems of great importance like $\mathrm{Ho}$ on $\mathrm{MgO} / \mathrm{Ag}(100)$ [38]. In particular, a reliable $a b$ initio scheme for the calculation of the electron-phonon coupling $[110,111]$ and its effect on the magnetic stability of single-adatoms is necessary in order to complement the knowledge gained on the electron-electron side. A second important ingredient that should be further considered in the future from an $a b$ initio viewpoint is the destabilizing effect of external particles, such as the tunneling electrons coming from an STM tip or X-ray photons of an XMCD measurement. In both cases, it is experimentally clear that the magnetic stability and spin lifetimes are severely affected by the intensity or flux of the incoming particles (see e.g., Refs [38, 42, 72].) First steps towards incorporating such effects into an ab initio scheme have been already taken in Ref. [54]: pending issues include the incorporation of SOC into the formalism as well as establishing a direct connection between the renormalization induced by the tunneling electrons and the magnetic stability of the adatom.

The conclusions drawn from the single-adatom case serve also as a solid platform for tackling more complex nanomagnets composed of several exchange-coupled magnetic atoms. This play- 
ground is particularly attractive given that several STM experiments have reported clear observations of stable magnetic moments in this type of composite system (see e.g., Refs. [112, 63, 113]). This strongly suggests that local fluctuation effects decrease with increasing cluster size, a feature that is in qualitative accordance with one main finding presented throughout the review, namely that the relative impact of the fluctuations becomes smaller the larger the magnetic moment gets. This general trend, however, is likely to be modified by characteristics associated to clusters, such as the appearance of optical modes in the spin-excitation spectrum (i.e. additional peaks in $\operatorname{Im} \chi_{\perp}(\omega)$, see e.g., Refs. [7, 52]) that would contribute to the fluctuations as seen from Eq. 6. On top of this, the impact of the fluctuations on the magnetic interactions among different adatoms remains to be fully explored and clarified.

Throughout the review we have also paid close attention to the time-scales of different spinrelaxation processes. We have studied two particular contributions, namely the longitudinal and transversal spin-relaxation times, providing their connection to the electronic structure of single-adatoms and, when possible, comparing the ab initio data to experimental measurements. In the case of $T_{\perp}$, we have shown that it is governed by atomic spin-excitations that induce the precessional motion of the magnetic moment, hence this relaxation time contributes to $T_{2}$ as commonly defined in the standard literature [96, 114]. Interestingly, there is yet another contribution to $T_{2}$ that has recently attracted a great interest in the single-adatom community, namely the adiabatic spin-decoherence time (see e.g., the review [23]). In fact, very recent EPR-based experiments have provided hard numbers for this quantity [22, 115], which is key for the potential use of single-adatoms as quantum devices. The ab initio calculation of this quantity is a challenging task for the future due to its pure quantum origin, which is not trivial to capture within the mean-field approach of DFT. Regarding the longitudinal spin-relaxation time, we have argued that, although currently available techniques cannot monitor the femtosecond regime of magnetic single adatoms, it is reasonable that this can be achieved in the near future, e.g., by employing STM-integrated ultrafast laser schemes [99, 100, 101], thus giving access to the ultrafast spin-dynamics described in this work.

As the last main topic, we have analyzed paramagnetic spin-excitations, i.e. fingerprints of magnetism in nonmagnetic adatoms, as well as means of detecting them in IETS experiments. Remarkably, a very recent measurement [116] of the conductance associated to a single $\mathrm{Pd}$ adatom deposited on $\mathrm{Pd}(111)$ has been interpreted as being strongly affected by paramagnon scattering, i.e., a potential indirect experimental observation of PSE. This opens up a promising range of applications for non-magnetic adatoms in nanotechnology, which encodes and manipulates information into excitation modes like PSE.

\section{Acknowledgments}

We gratefully acknowledge discussions and common work with B. Schweflinghaus, as well as fruitful scientific exchange with F. Guimarães, A. Eiguren, N. Lorente and P. H. Dederichs on the theory side, and J. Hermenau and J. Wiebe on the experimental side. This work has been supported by the Helmholtz Gemeinschaft Deutscher-Young Investigators Group Program No. VH-NG-717 (Functional Nanoscale Structure and Probe Simulation Laboratory), the Impuls 
und Vernetzungsfonds der Helmholtz-Gemeinschaft Postdoc Programme, and funding from the European Research Council (ERC) under the European Union's Horizon 2020 research and innovation programme (ERC-consolidator grant 681405 DYNASORE). We also acknowledge the computing time granted by the JARA-HPC Vergabegremium and provided on the JARAHPC Partition part of the supercomputer JURECA at Forschungszentrum Jülich.

\section{Appendices}

\section{A Longitudinal Bloch equation}

The Bloch equation for the longitudinal change of the magnetization under the effect of a timedependent perturbation $H_{1}(t)$ along the longitudinal direction can be written as [96]

$$
\frac{\mathrm{d} m_{z}(t)}{\mathrm{d} t}=\frac{\chi_{0}^{\mathrm{Bl}} H_{1}(t)-m_{z}(t)}{T_{\|}}
$$

with $\chi_{0}^{\mathrm{Bl}}$ a static spin-susceptibility. The above equation describes how $m_{z}(t)$ comes back to equilibrium with a characteristic relaxation time $T_{\|}$after being perturbed by $H_{1}(t)$. Using $f(t)=\int \mathrm{d} \omega f(\omega) e^{-\mathrm{i} \omega t}$ for both $m_{z}(t)$ and $H_{1}(t)$ we can write Eq. 28 in the frequency domain,

$$
\begin{aligned}
& m_{z}(\omega)\left(-\mathrm{i} \omega T_{\|}+1\right)=\chi_{0} H_{1}(\omega) \Rightarrow \\
& \frac{m_{z}(\omega)}{H_{1}(\omega)} \equiv \chi^{\mathrm{Bl}}(\omega)=\frac{\chi_{0}^{\mathrm{Bl}}}{1-\mathrm{i} \omega T_{\|}},
\end{aligned}
$$

where $\chi^{\mathrm{Bl}}(\omega)$ is the enhanced spin-susceptibility. The real and imaginary parts of the above equation read

$$
\begin{aligned}
& \operatorname{Re} \chi^{\mathrm{Bl}}(\omega)=\frac{\chi_{0}^{\mathrm{Bl}}}{1+\left(\omega T_{\|}\right)^{2}}, \\
& \operatorname{Im} \chi^{\mathrm{Bl}}(\omega)=\frac{\chi_{0}^{\mathrm{Bl}} \omega T_{\|}}{1+\left(\omega T_{\|}\right)^{2}} .
\end{aligned}
$$

Next, we consider the Taylor expansion of the KS spin-susceptibility (see Eq. 15 in the main text),

$$
\chi_{\|}^{\mathrm{KS}}(\omega) \simeq \rho_{\mathrm{F}}-\mathrm{i} n_{\mathrm{e}-\mathrm{h}} \omega .
$$

The first-order expansion coefficient $n_{\mathrm{e}-\mathrm{h}}=\pi\left(\rho_{\mathrm{F}, \uparrow}^{2}+\rho_{\mathrm{F}, \downarrow}^{2}\right) / 2$ has been calculated in the Supplemental Material of Ref. [77] . Inserting $\chi_{\|}^{\mathrm{KS}}(\omega)$ of Eq. 32 into the definition of the TDDFT enhanced spin-susceptibility $\chi(\omega)$ (see Eq. 7 of the main text), the imaginary part $\operatorname{Im} \chi_{\|}(\omega)$ can be cast in the following way,

$$
\begin{aligned}
\operatorname{Im} \chi_{\|}(\omega) & =\frac{n_{\mathrm{e}-\mathrm{h}} \omega}{\left(1-U_{\|} \rho_{\mathrm{F}}\right)^{2}} \frac{1}{1+\left(\frac{U_{\|} n_{\mathrm{e}-\mathrm{h}} \omega}{1-U_{\|} \rho_{\mathrm{F}}}\right)^{2}} \\
& =\frac{\omega T_{\|}}{\left(1-U_{\|} \rho_{\mathrm{F}}\right) U_{\|}} \frac{1}{1+\left(\omega T_{\|}\right)^{2}}=\frac{\chi(0)}{U_{\|} \rho_{\mathrm{F}}} \frac{\omega T_{\|}}{1+\left(\omega T_{\|}\right)^{2}},
\end{aligned}
$$


where in the last step we used the expression for the static spin-susceptibility $\chi_{\|}(0)=\rho_{\mathrm{F}} /(1-$ $\left.U_{\|} \rho_{\mathrm{F}}\right)$ and we defined

$$
T_{\|}=\frac{U_{\|} n_{\mathrm{e}-\mathrm{h}}}{1-U_{\|} \rho_{\mathrm{F}}}
$$

which is the result quoted in the main text in Eq. 16.

\section{B Transverse relaxation within the Landau-Lifshitz-Gilbert equa- tion}

We consider the Landau-Lifshitz-Gilbert (LLG) equation describing the damped precessional motion of a magnetic moment placed in a static external magnetic field that has been perturbed by a time-dependent transverse magnetic field:

$$
\frac{\mathrm{d} \mathbf{m}}{\mathrm{d} t}=-\gamma \mathbf{m} \times \mathbf{B}^{\mathrm{ext}}+\eta \frac{\mathbf{m}}{m_{0}} \times \frac{\mathrm{d} \mathbf{m}}{\mathrm{d} t},
$$

with $\mathbf{B}^{\text {ext }}=B_{0} \hat{\mathbf{z}}+\mathbf{b}(t)$ and $\mathbf{b}(t)=b_{x}(t) \hat{\mathbf{x}}+b_{y}(t) \hat{\mathbf{y}}$. Here $B_{0}$ is the static part of the external field, and $\mathbf{b}(t)$ a small transverse perturbing field, $|\mathbf{b}(t)| \ll B_{0}$. The precession rate in Eq. 35 is set by $\gamma$, while the relaxation is controlled by $\eta$, namely the damping term. Writing $\mathbf{m}(t)=m_{0} \hat{\mathbf{z}}+\mathbf{m}(t)$ with $\mathbf{m}(t)=m_{x}(t) \hat{\mathbf{x}}+m_{y}(t) \hat{\mathbf{y}}$, and linearizing the LLG equation yields the following equation of motion for the transverse components of the magnetization:

$$
\begin{aligned}
\frac{\mathrm{d} m_{x}}{\mathrm{~d} t} & =-\gamma B_{0} m_{y}+\gamma m_{0} b_{y}-\eta \frac{\mathrm{d} m_{y}}{\mathrm{~d} t} \\
\frac{\mathrm{d} m_{y}}{\mathrm{~d} t} & =-\gamma m_{0} b_{x}+\gamma B_{0} m_{x}+\eta \frac{\mathrm{d} m_{x}}{\mathrm{~d} t} .
\end{aligned}
$$

To illustrate the relaxation dynamics, consider a static external field $(\mathbf{b}(t)=0)$ and a small tilt of the magnetic moment at some initial time: $\mathbf{m}(t=0)=m_{0}(\cos \theta \hat{\mathbf{z}}+\sin \theta \hat{\mathbf{x}}) \approx m_{0} \hat{\mathbf{z}}+\Delta m \hat{\mathbf{x}}$. Since the expected solution is a damped precession that relaxes towards the direction of the static magnetic field, we use the following ansatz corresponding to a circular precession that decays in time with a transverse relaxation time $T_{\perp}$ :

$$
\begin{aligned}
& m_{x}(t)=\Delta m e^{-t / T_{\perp}} \cos \left(\omega_{0} t\right), \\
& m_{y}(t)=\Delta m e^{-t / T_{\perp}} \sin \left(\omega_{0} t\right) .
\end{aligned}
$$

Plugging the above ansatz back into the LLG Eqs. 36 and 37 we get

$$
\begin{aligned}
\frac{1}{T_{\perp}} \cos \left(\omega_{0} t\right)+\omega_{0} \sin \left(\omega_{0} t\right) & =\eta \omega_{0} \cos \left(\omega_{0} t\right)+\left(\gamma B_{0}-\frac{\eta}{T_{\perp}}\right) \sin \left(\omega_{0} t\right), \\
-\frac{1}{T_{\perp}} \sin \left(\omega_{0} t\right)+\omega_{0} \cos \left(\omega_{0} t\right) & =-\eta \omega_{0} \sin \left(\omega_{0} t\right)+\left(\gamma B_{0}-\frac{\eta}{T_{\perp}}\right) \sin \left(\omega_{0} t\right) .
\end{aligned}
$$

The above equations can only be satisfied if the coefficients in front of the time-dependent sines and cosines match. We then have (both equations give the same pair of relations)

$$
\begin{gathered}
\frac{1}{T_{\perp}}=\eta \omega_{0}, \\
\omega_{0}=\frac{\gamma B_{0}}{1+\eta^{2}} .
\end{gathered}
$$


Importantly, Eq. 42 shows that the transverse relaxation time is given by the product between the damping term $\eta$ and the characteristic frequency $\omega_{0}$.

We next turn to calculate the transverse dynamic spin-susceptibility within the LLG model. For this, we consider the following Fourier transforms,

$$
\mathbf{b}(t)=\int \frac{\mathrm{d} \omega}{2 \pi} e^{-\mathrm{i} \omega t} \mathbf{b}(\omega), \mathbf{m}(t)=\int \frac{\mathrm{d} \omega}{2 \pi} e^{-\mathrm{i} \omega t} \mathbf{m}(\omega) .
$$

Inserting the above expressions into the linearized equations 36 and 37 we obtain in frequency space

$$
\begin{aligned}
& -\mathrm{i} \omega m_{x}(\omega)=+\gamma m_{0} b_{y}(\omega)+\left(\mathrm{i} \eta \omega-\gamma B_{0}\right) m_{y}(\omega), \\
& -\mathrm{i} \omega m_{y}(\omega)=-\gamma m_{0} b_{x}(\omega)+\left(\gamma B_{0}-\mathrm{i} \eta \omega\right) m_{x}(\omega) .
\end{aligned}
$$

The above can be simplified by considering the circular components $m_{ \pm}=m_{x} \pm \mathrm{i} m_{y}$, yielding

$$
\begin{gathered}
\omega m_{ \pm}(\omega)=\gamma_{ \pm}\left(m_{0} b_{ \pm}(\omega)-B_{0} m_{ \pm}(\omega)\right), \Rightarrow \\
\Lambda_{ \pm}(\omega) m_{ \pm}(\omega)=b_{ \pm}(\omega),
\end{gathered}
$$

with $b_{ \pm}=b_{x} \pm \mathrm{i} b_{y}, \gamma_{ \pm}= \pm \gamma /(1 \mp \mathrm{i} \eta)$ and

$$
\Lambda_{ \pm}(\omega)=\frac{B_{0}}{m_{0}}+\frac{\omega}{\gamma_{ \pm} m_{0}} .
$$

It is apparent from Eq. 47 that the transverse spin-susceptibility can be obtained from the inverse of $\Lambda_{ \pm}(\omega)$ defined above. After some algebra and picking the minus sign in Eq. 48 one obtains

$$
\chi_{ \pm}^{\mathrm{LLG}}(\omega)=\left(\Lambda_{-}(\omega)\right)^{-1}=\frac{m_{0} \omega_{0}}{B_{0}} \cdot \frac{-\omega+\left(1+\eta^{2}\right) \omega_{0}+\mathrm{i} \eta \omega}{\left(\omega-\omega_{0}\right)^{2}+\left(\eta \omega_{0}\right)^{2}} .
$$

The density of spin-excitations in the LLG model are thus described by a skewed Lorentzian in $\omega$ :

$$
\operatorname{Im} \chi_{ \pm}^{\mathrm{LLG}}(\omega)=\frac{m_{0} \omega_{0}}{B_{0}} \frac{\eta \omega}{\left(\omega-\omega_{0}\right)^{2}+\left(\eta \omega_{0}\right)^{2}} .
$$

The resonance frequency of the above function takes place at

$$
\frac{\mathrm{d}}{\mathrm{d} \omega} \operatorname{Im} \chi_{ \pm}^{\mathrm{LLG}}(\omega)=0 \Rightarrow \omega_{\text {res }}=\sqrt{1+\eta^{2}} \omega_{0}
$$

while the FWHM amounts to

$$
\Gamma=2 \eta \omega_{0} \frac{\sqrt{2+3 \eta^{2}+2 \sqrt{1+\eta^{2}}}}{1+\sqrt{1+\eta^{2}}} \simeq 2 \eta \omega_{0} .
$$

We note that the above approximation is exact in the $\eta \rightarrow 0$ limit and involves only a $\sim 10 \%$ relative error for $\eta=1$, which is by far the maximum value that damping can get for single adatoms; for most of the elements analyzed in the main text we have $\eta \lesssim 0.5$ [81], so the approximation of Eq. 52 is indeed very good. Then, comparing Eq. 52 to Eq. 42 we arrive to the relation between the FWHM and the transverse relaxation time quoted in the main text:

$$
T_{\perp}=\frac{2}{\Gamma}
$$




\section{References}

[1] A. J. Heinrich, J. A. Gupta, C. P. Lutz, and D. M. Eigler. Single-Atom Spin-Flip Spectroscopy. Science, 306(5695):466-469, October 2004.

[2] Cyrus F. Hirjibehedin, Christopher P. Lutz, and Andreas J. Heinrich. Spin coupling in engineered atomic structures. Science, 312(5776):1021-1024, 2006.

[3] Cyrus F. Hirjibehedin, Chiung-Yuan Lin, Alexander F. Otte, Markus Ternes, Christopher P. Lutz, Barbara A. Jones, and Andreas J. Heinrich. Large Magnetic Anisotropy of a Single Atomic Spin Embedded in a Surface Molecular Network. Science, 317(5842):11991203, August 2007.

[4] T Balashov, T Schuh, AF Takács, A Ernst, S Ostanin, J Henk, I Mertig, P Bruno, T Miyamachi, S Suga, et al. Magnetic anisotropy and magnetization dynamics of individual atoms and clusters of fe and co on pt (111). Phys. Rev. Lett., 102(25):257203, 2009.

[5] Nicolás Lorente and Jean-Pierre Gauyacq. Efficient spin transitions in inelastic electron tunneling spectroscopy. Phys. Rev. Lett., 103:176601, Oct 2009.

[6] J. Fernández-Rossier. Theory of single-spin inelastic tunneling spectroscopy. Phys. Rev. Lett., 102:256802, Jun 2009.

[7] S. Lounis, A. T. Costa, R. B. Muniz, and D. L. Mills. Dynamical Magnetic Excitations of Nanostructures from First Principles. Physical Review Letters, 105(18):187205, October 2010.

[8] A. A. Khajetoorians, S. Lounis, B. Chilian, A. T. Costa, L. Zhou, D. L. Mills, J. Wiebe, and R. Wiesendanger. Itinerant nature of atom-magnetization excitation by tunneling electrons. Phys. Rev. Lett., 106:037205, Jan 2011.

[9] A. A. Khajetoorians, T. Schlenk, B. Schweflinghaus, M. dos Santos Dias, M. Steinbrecher, M. Bouhassoune, S. Lounis, J. Wiebe, and R. Wiesendanger. Spin excitations of individual fe atoms on pt(111): Impact of the site-dependent giant substrate polarization. Phys. Rev. Lett., 111:157204, Oct 2013.

[10] F. Donati, Q. Dubout, G. Autès, F. Patthey, F. Calleja, P. Gambardella, O. V. Yazyev, and H. Brune. Magnetic moment and anisotropy of individual co atoms on graphene. Phys. Rev. Lett., 111:236801, December 2013.

[11] Markus Ternes. Spin excitations and correlations in scanning tunneling spectroscopy. New Journal of Physics, 17(6):063016, 2015.

[12] A. A. Khajetoorians, M. Steinbrecher, M. Ternes, M. Bouhassoune, M. dos Santos Dias, S. Lounis, J. Wiebe, and R. Wiesendanger. Tailoring the chiral magnetic interaction between two individual atoms. Nature Communications, 7:10620, February 2016.

[13] Filipe S. M. Guimarães, Manuel dos Santos Dias, Benedikt Schweflinghaus, and Samir Lounis. Engineering elliptical spin-excitations by complex anisotropy fields in fe adatoms and dimers on cu(111). Phys. Rev. B, 96:144401, Oct 2017. 
[14] Jan Hermenau, Markus Ternes, Manuel Steinbrecher, Roland Wiesendanger, and Jens Wiebe. Long spin-relaxation times in a transition-metal atom in direct contact to a metal substrate. Nano Letters, 18(3):1978-1983.

[15] Focko Meier, Lihui Zhou, Jens Wiebe, and Roland Wiesendanger. Revealing magnetic interactions from single-atom magnetization curves. Science, 320(5872):82-86, 2008.

[16] Lihui Zhou, Jens Wiebe, Samir Lounis, Elena Vedmedenko, Focko Meier, Stefan Blügel, Peter H. Dederichs, and Roland Wiesendanger. Strength and directionality of surface Ruderman-Kittel-Kasuya-Yosida interaction mapped on the atomic scale. Nature Physics, 6(3):187-191, March 2010.

[17] Anna Stróżecka, Asier Eiguren, and Jose Ignacio Pascual. Quasiparticle interference around a magnetic impurity on a surface with strong spin-orbit coupling. Phys. Rev. Lett., 107:186805, Oct 2011.

[18] Alexander Ako Khajetoorians, Jens Wiebe, Bruno Chilian, Samir Lounis, Stefan Blügel, and Roland Wiesendanger. Atom-by-atom engineering and magnetometry of tailored nanomagnets. Nat. Phys., 8(6):497-503, 2012.

[19] Jenny C. Oberg, M. Reyes Calvo, Fernando Delgado, Mara Moro-Lagares, David Serrate, David Jacob, Joaqun Fernández-Rossier, and Cyrus F. Hirjibehedin. Control of single-spin magnetic anisotropy by exchange coupling. Nature Nanotechnology, 9(1):64-68, January 2014.

[20] Shichao Yan, Deung-Jang Choi, Burgess Jacob A. J., Steffen Rolf-Pissarczyk, and Sebastian Loth. Control of quantum magnets by atomic exchange bias. Nat Nano, 10(1):40-45, January 2015.

[21] Benjamin Bryant, Ranko Toskovic, Alejandro Ferrón, Jos L. Lado, Anna Spinelli, Joaqun Fernández-Rossier, and Alexander F. Otte. Controlled complete suppression of single-atom inelastic spin and orbital cotunneling. Nano Letters, 15(10):6542-6546, 2015.

[22] Susanne Baumann, William Paul, Taeyoung Choi, Christopher P. Lutz, Arzhang Ardavan, and Andreas J. Heinrich. Electron paramagnetic resonance of individual atoms on a surface. Science, 350(6259):417-420, October 2015.

[23] F. Delgado and J. Fernández-Rossier. Spin decoherence of magnetic atoms on surfaces. Progress in Surface Science, 92(1):40-82, February 2017.

[24] Shuai-Hua Ji, Tong Zhang, Ying-Shuang Fu, Xi Chen, Xu-Cun Ma, Jia Li, Wen-Hui Duan, Jin-Feng Jia, and Qi-Kun Xue. High-resolution scanning tunneling spectroscopy of magnetic impurity induced bound states in the superconducting gap of pb thin films. Phys. Rev. Lett., 100:226801, Jun 2008.

[25] Bernd Braunecker and Pascal Simon. Interplay between classical magnetic moments and superconductivity in quantum one-dimensional conductors: Toward a self-sustained topological majorana phase. Phys. Rev. Lett., 111:147202, Oct 2013. 
[26] B. W. Heinrich, L. Braun, J. I. Pascual, and K. J. Franke. Protection of excited spin states by a superconducting energy gap. Nature Physics, 9(12):765-768, 2013.

[27] M. M. Vazifeh and M. Franz. Self-organized topological state with majorana fermions. Phys. Rev. Lett., 111:206802, Nov 2013.

[28] Stevan Nadj-Perge, Ilya K. Drozdov, Jian Li, Hua Chen, Sangjun Jeon, Jungpil Seo, Allan H. MacDonald, B. Andrei Bernevig, and Ali Yazdani. Observation of Majorana fermions in ferromagnetic atomic chains on a superconductor. Science, 346(6209):602607, October 2014.

[29] Michael Ruby, Falko Pientka, Yang Peng, Felix von Oppen, Benjamin W. Heinrich, and Katharina J. Franke. End states and subgap structure in proximity-coupled chains of magnetic adatoms. Phys. Rev. Lett., 115:197204, November 2015.

[30] Michael Ruby, Yang Peng, Felix von Oppen, Benjamin W. Heinrich, and Katharina J. Franke. Orbital picture of yu-shiba-rusinov multiplets. Phys. Rev. Lett., 117:186801, October 2016.

[31] V. Madhavan, W. Chen, T. Jamneala, M. F. Crommie, and N. S. Wingreen. Tunneling into a single magnetic atom: Spectroscopic evidence of the kondo resonance. Science, 280(5363):567-569, 1998.

[32] N. Néel, J. Kröger, R. Berndt, T. O. Wehling, A. I. Lichtenstein, and M. I. Katsnelson. Controlling the kondo effect in cocu $_{n}$ clusters atom by atom. Phys. Rev. Lett., 101:266803, December 2008.

[33] Alexander F Otte, Markus Ternes, Kirsten von Bergmann, Sebastian Loth, Harald Brune, Christopher P Lutz, Cyrus F Hirjibehedin, and Andreas J Heinrich. The role of magnetic anisotropy in the kondo effect. Nat. Phys., 4(11):847-850, 2008.

[34] AF Otte, M Ternes, S Loth, CP Lutz, CF Hirjibehedin, and AJ Heinrich. Spin excitations of a kondo-screened atom coupled to a second magnetic atom. Phys. Rev. Lett., 103(10):107203, 2009.

[35] Markus Ternes, Andreas J. Heinrich, and Wolf-Dieter Schneider. Spectroscopic manifestations of the Kondo effect on single adatoms. Journal of Physics: Condensed Matter, 21(5):053001, 2009.

[36] F. Delgado, C.F. Hirjibehedin, and J. Fernández-Rossier. Consequences of kondo exchange on quantum spins. Surf. Sci., 630(0):337-342, 2014.

[37] Kirsten von Bergmann, Markus Ternes, Sebastian Loth, Christopher P. Lutz, and Andreas J. Heinrich. Spin polarization of the split kondo state. Phys. Rev. Lett., 114:076601, 2015 .

[38] F. Donati, S. Rusponi, S. Stepanow, C. Wäckerlin, A. Singha, L. Persichetti, R. Baltic, K. Diller, F. Patthey, E. Fernandes, J. Dreiser, Ž. Šljivančanin, K. Kummer, C. Nistor, P. Gambardella, and H. Brune. Magnetic remanence in single atoms. Science, 352(6283):318-321, 2016. 
[39] Harald Brune and Pietro Gambardella. Magnetism of individual atoms adsorbed on surfaces. Surf. Sci., 603(10-12):1812-1830, 2009. Special Issue of Surface Science dedicated to Prof. Dr. Dr. h.c. mult. Gerhard Ertl, Nobel-Laureate in Chemistry 2007.

[40] Alexander F. Otte, Markus Ternes, Kirsten von Bergmann, Sebastian Loth, Harald Brune, Christopher P. Lutz, Cyrus F. Hirjibehedin, and Andreas J. Heinrich. The role of magnetic anisotropy in the Kondo effect. Nature Physics, 4(11):847-850, November 2008.

[41] Sebastian Loth, Markus Etzkorn, Christopher P. Lutz, D. M. Eigler, and Andreas J. Heinrich. Measurement of Fast Electron Spin Relaxation Times with Atomic Resolution. Science, 329(5999):1628-1630, September 2010.

[42] Fabian D. Natterer, Kai Yang, William Paul, Philip Willke, Taeyoung Choi, Thomas Greber, Andreas J. Heinrich, and Christopher P. Lutz. Reading and writing single-atom magnets. Nature, 543(7644):226-228, March 2017.

[43] A. Oswald, R. Zeller, and P. H. Dederichs. Giant Moments in Palladium. Physical Review Letters, 56(13):1419-1422, March 1986.

[44] K. Wildberger, V. S. Stepanyuk, P. Lang, R. Zeller, and P. H. Dederichs. Magnetic Nanostructures: 4 d Clusters on $\operatorname{Ag}(001)$. Physical Review Letters, 75(3):509-512, July 1995.

[45] P. Lang, V. S. Stepanyuk, K. Wildberger, R. Zeller, and P. H. Dederichs. Local moments of $3 \mathrm{~d}, 4 \mathrm{~d}$, and $5 \mathrm{~d}$ atoms at $\mathrm{Cu}$ and $\mathrm{Ag}(001)$ surfaces. Solid State Communications, 92(9):755-759, December 1994.

[46] Cyrus F. Hirjibehedin, Christopher P. Lutz, and Andreas J. Heinrich. Spin Coupling in Engineered Atomic Structures. Science, 312(5776):1021-1024, May 2006.

[47] Nicols Lorente and Jean-Pierre Gauyacq. Efficient Spin Transitions in Inelastic Electron Tunneling Spectroscopy. Physical Review Letters, 103(17):176601, October 2009.

[48] Mats Persson. Theory of inelastic electron tunneling from a localized spin in the impulsive approximation. Phys. Rev. Lett., 103:050801, July 2009.

[49] J. Fransson. Spin Inelastic Electron Tunneling Spectroscopy on Local Spin Adsorbed on Surface. Nano Letters, 9(6):2414-2417, June 2009.

[50] Bjrn Sothmann and Jrgen Knig. Nonequilibrium current and noise in inelastic tunneling through a magnetic atom. New Journal of Physics, 12(8):083028, 2010.

[51] Jean-Pierre Gauyacq, Nicolás Lorente, and Frederico Dutilh Novaes. Excitation of local magnetic moments by tunneling electrons. Prog. Surf. Sci., 87(5):63-107, 2012.

[52] S. Lounis, A. T. Costa, R. B. Muniz, and D. L. Mills. Theory of local dynamical magnetic susceptibilities from the Korringa-Kohn-Rostoker Green function method. Physical Review B, 83(3):035109, January 2011.

[53] M. dos Santos Dias, B. Schweflinghaus, S. Blügel, and S. Lounis. Relativistic dynamical spin excitations of magnetic adatoms. Phys. Rev. B, 91:075405, Feb 2015. 
[54] Benedikt Schweflinghaus, Manuel dos Santos Dias, Antonio T. Costa, and Samir Lounis. Renormalization of electron self-energies via their interaction with spin excitations: A first-principles investigation. Phys. Rev. B, 89:235439, Jun 2014.

[55] P. Gambardella, S. Rusponi, M. Veronese, S. S. Dhesi, C. Grazioli, A. Dallmeyer, I. Cabria, R. Zeller, P. H. Dederichs, K. Kern, C. Carbone, and H. Brune. Giant Magnetic Anisotropy of Single Cobalt Atoms and Nanoparticles. Science, 300(5622):1130-1133, May 2003.

[56] B. Bryant, A. Spinelli, J. J. T. Wagenaar, M. Gerrits, and A. F. Otte. Local control of single atom magnetocrystalline anisotropy. Phys. Rev. Lett., 111:127203, Sep 2013.

[57] Ileana G. Rau, Susanne Baumann, Stefano Rusponi, Fabio Donati, Sebastian Stepanow, Luca Gragnaniello, Jan Dreiser, Cinthia Piamonteze, Frithjof Nolting, Shruba Gangopadhyay, Oliver R. Albertini, Roger M. Macfarlane, Christopher P. Lutz, Barbara A. Jones, Pietro Gambardella, Andreas J. Heinrich, and Harald Brune. Reaching the magnetic anisotropy limit of a 3d metal atom. Science, 344(6187):988-992, May 2014.

[58] Focko Meier, Lihui Zhou, Jens Wiebe, and Roland Wiesendanger. Revealing Magnetic Interactions from Single-Atom Magnetization Curves. Science, 320(5872):82-86, April 2008 .

[59] Alexander Ako Khajetoorians, Jens Wiebe, Bruno Chilian, Samir Lounis, Stefan Blügel, and Roland Wiesendanger. Atom-by-atom engineering and magnetometry of tailored nanomagnets. Nature Physics, 8(6):497-503, June 2012.

[60] J. Honolka, K. Kuhnke, L. Vitali, A. Enders, K. Kern, S. Gardonio, C. Carbone, S. R. Krishnakumar, P. Bencok, S. Stepanow, and P. Gambardella. Absence of local magnetic moments in ru and rh impurities and clusters on ag(100) and pt(997). Physical Review B, 76(14):144412, October 2007.

[61] Toshio Miyamachi, Tobias Schuh, Tobias Märkl, Christopher Bresch, Timofey Balashov, Alexander Stöhr, Christian Karlewski, Stephan André, Michael Marthaler, Martin Hoffmann, Matthias Geilhufe, Sergey Ostanin, Wolfram Hergert, Ingrid Mertig, Gerd Schön, Arthur Ernst, and Wulf Wulfhekel. Stabilizing the magnetic moment of single holmium atoms by symmetry. Nature, 503(7475):242-246, November 2013.

[62] C. Hübner, B. Baxevanis, A. A. Khajetoorians, and D. Pfannkuche. Symmetry effects on the spin switching of adatoms. Phys. Rev. B, 90(15):155134, October 2014.

[63] Alexander Ako Khajetoorians, Benjamin Baxevanis, Christoph Hübner, Tobias Schlenk, Stefan Krause, Tim Oliver Wehling, Samir Lounis, Alexander Lichtenstein, Daniela Pfannkuche, Jens Wiebe, and Roland Wiesendanger. Current-Driven Spin Dynamics of Artificially Constructed Quantum Magnets. Science, 339(6115):55-59, January 2013.

[64] Herbert B. Callen and Theodore A. Welton. Irreversibility and generalized noise. Phys. Rev., 83:34-40, Jul 1951.

[65] T. Moriya. Spin Fluctuations in Itinerant Electron Magnetism. Springer, Berlin, 1985. 
[66] Yoshinori Takahashi. On the origin of the curie-weiss law of the magnetic susceptibility in itinerant electron ferromagnetism. Journal of the Physical Society of Japan, 55(10):3553$3573,1986$.

[67] G. G. Lonzarich and L. Taillefer. Effect of spin fluctuations on the magnetic equation of state of ferromagnetic or nearly ferromagnetic metals. Journal of Physics C: Solid State Physics, 18(22):4339, August 1985.

[68] M. Shimizu. Itinerant electron magnetism. Reports on Progress in Physics, 44(4):329, 1981.

[69] A. Aguayo, I. I. Mazin, and D. J. Singh. Why ni3al is an itinerant ferromagnet but ni3ga is not. Physical Review Letters, 92(14):147201, April 2004.

[70] L. Ortenzi, I. I. Mazin, P. Blaha, and L. Boeri. Accounting for spin fluctuations beyond local spin density approximation in the density functional theory. Physical Review B, 86(6):064437, August 2012.

[71] Sebastian Loth, Christopher P Lutz, and Andreas J Heinrich. Spin-polarized spin excitation spectroscopy. New Journal of Physics, 12(12):125021, 2010.

[72] William Paul, Kai Yang, Susanne Baumann, Niklas Romming, Taeyoung Choi, Christopher P. Lutz, and Andreas J. Heinrich. Control of the millisecond spin lifetime of an electrically probed atom. Nature Physics, 13(4):403-407, April 2017.

[73] S. Doniach. Theory of inelastic neutron scattering in nearly ferromagnetic metals. Proceedings of the Physical Society, 91(1):86, 1967.

[74] R. Doubble, S. M. Hayden, Pengcheng Dai, H. A. Mook, J. R. Thompson, and C. D. Frost. Direct observation of paramagnons in palladium. Phys. Rev. Lett., 105:027207, Jul 2010.

[75] J. B. Staunton, J. Poulter, B. Ginatempo, E. Bruno, and D. D. Johnson. Spin fluctuations in nearly magnetic metals from ab initio dynamical spin susceptibility calculations: Application to Pd and Cr95v5. Physical Review B, 62(2):1075-1082, July 2000.

[76] G. F. Giuliani and G. Vignale. Quantum Theory of the Electron Liquid. Cambridge University Press, 2005.

[77] Julen Ibañez-Azpiroz, Manuel dos Santos Dias, Benedikt Schweflinghaus, Stefan Blügel, and Samir Lounis. Tuning paramagnetic spin excitations of single adatoms. Phys. Rev. Lett., 119:017203, Jul 2017.

[78] Erich Runge and E. K. U. Gross. Density-functional theory for time-dependent systems. Phys. Rev. Lett., 52:997-1000, Mar 1984.

[79] M. Petersilka, U. J. Gossmann, and E. K. U. Gross. Excitation energies from timedependent density-functional theory. Phys. Rev. Lett., 76:1212-1215, Feb 1996.

[80] Samir Lounis, Manuel dos Santos Dias, and Benedikt Schweflinghaus. Transverse dynamical magnetic susceptibilities from regular static density functional theory: Evaluation of damping and $g$ shifts of spin excitations. Phys. Rev. B, 91:104420, Mar 2015. 
[81] Julen Ibañez-Azpiroz, Manuel dos Santos Dias, Stefan Blügel, and Samir Lounis. ZeroPoint Spin-Fluctuations of Single Adatoms. Nano Letters, 16(7):4305-4311, July 2016.

[82] N. Papanikolaou, R. Zeller, and P. H. Dederichs. Conceptual improvements of the KKR method. Journal of Physics: Condensed Matter, 14(11):2799, March 2002.

[83] S. H. Vosko, L. Wilk, and M. Nusair. Accurate spin-dependent electron liquid correlation energies for local spin density calculations: a critical analysis. Canadian Journal of Physics, 58(8):1200-1211, August 1980.

[84] Julen Ibañez-Azpiroz, Manuel dos Santos Dias, Stefan Blügel, and Samir Lounis. Longitudinal and transverse spin relaxation times of magnetic single adatoms: An ab initio analysis. Phys. Rev. B, 96:144410, Oct 2017.

[85] A. L. Wysocki, V. N. Valmispild, A. Kutepov, S. Sharma, J. K. Dewhurst, E. K. U. Gross, A. I. Lichtenstein, and V. P. Antropov. Spin-density fluctuations and the fluctuationdissipation theorem in 3d ferromagnetic metals. Phys. Rev. B, 96:184418, Nov 2017.

[86] H. Beckmann, R. Schäfer, Wenqi Li, and G. Bergmann. First observation of a fully magnetic 4d impurity on the surface of au. EPL (Europhysics Letters), 33(7):563, March 1996.

[87] V. Madhavan, W. Chen, T. Jamneala, M. F. Crommie, and N. S. Wingreen. Tunneling into a Single Magnetic Atom: Spectroscopic Evidence of the Kondo Resonance. Science, 280(5363):567-569, April 1998.

[88] H. C. Manoharan, C. P. Lutz, and D. M. Eigler. Quantum mirages formed by coherent projection of electronic structure. Nature, 403(6769):512-515, February 2000.

[89] L. Limot, E. Pehlke, J. Kröger, and R. Berndt. Surface-state localization at adatoms. Phys. Rev. Lett., 94:036805, Jan 2005.

[90] T.L. Gilbert. A phenomenological theory of damping in ferromagnetic materials. IEEE Transactions on Magnetics, 40(6):3443-3449, November 2004.

[91] R. Wieser. Comparison of quantum and classical relaxation in spin dynamics. Phys. Rev. Lett., 110:147201, Apr 2013.

[92] A. A. Clerk, M. H. Devoret, S. M. Girvin, Florian Marquardt, and R. J. Schoelkopf. Introduction to quantum noise, measurement, and amplification. Reviews of Modern Physics, 82(2):1155-1208, April 2010.

[93] M. Weinert, R. E. Watson, and J. W. Davenport. Total-energy differences and eigenvalue sums. Phys. Rev. B, 32:2115-2119, Aug 1985.

[94] Giulia E. Pacchioni, Luca Gragnaniello, Fabio Donati, Marina Pivetta, Gabriel Autès, Oleg V. Yazyev, Stefano Rusponi, and Harald Brune. Multiplet features and magnetic properties of fe on $\mathrm{cu}(111)$ : From single atoms to small clusters. Phys. Rev. B, 91:235426, Jun 2015. 
[95] R. Schafer and G. Bergmann. Nb atoms on the surface of Ag, an unexpected kondo system. Solid State Communications, 98(1):45-48, April 1996.

[96] Robert M. White. Quantum Theory of Magnetism: Magnetic Properties of Materials. Springer, 2007.

[97] Jrgen Kübler. Theory of Itinerant Electron Magnetism. International Series of Monographs on Physics. Oxford University Press, Oxford, New York, June 2009.

[98] J. F. Janak. Uniform susceptibilities of metallic elements. Physical Review B, 16(1), July 1977.

[99] Michael Krüger, Markus Schenk, and Peter Hommelhoff. Attosecond control of electrons emitted from a nanoscale metal tip. Nature, 475(7354):78-81, July 2011.

[100] Tyler L. Cocker, Vedran Jelic, Manisha Gupta, Sean J. Molesky, Jacob A. J. Burgess, Glenda De Los Reyes, Lyubov V. Titova, Ying Y. Tsui, Mark R. Freeman, and Frank A. Hegmann. An ultrafast terahertz scanning tunnelling microscope. Nature Photonics, 7(8):620-625, August 2013.

[101] Tyler L. Cocker, Dominik Peller, Ping Yu, Jascha Repp, and Rupert Huber. Tracking the ultrafast motion of a single molecule by femtosecond orbital imaging. Nature, 539(7628):263-267, November 2016.

[102] B. Lazarovits, L. Szunyogh, and P. Weinberger. Fully relativistic calculation of magnetic properties of fe, co, and ni adclusters on ag(100). Phys. Rev. B, 65:104441, Mar 2002.

[103] H. Beckmann and G. Bergmann. Magnetism of Rh and Ru atoms, clusters, and monolayers on Au and Ag surfaces. Physical Review B, 55(21):14350-14359, June 1997.

[104] For externally applied $B=517 \mathrm{~T}$ the magnetic moment of $\mathrm{Ni}$ is $m=0.29 \mu_{B}$, while a Fe adatom placed as a second nearest neighbor induces a magnetic moment on $\mathrm{Ni}$ of $m=0.51$ $\mu_{B}$.

[105] M. Steinbrecher, A. Sonntag, M. dos Santos Dias, M. Bouhassoune, S. Lounis, J. Wiebe, R. Wiesendanger, and A. A. Khajetoorians. Absence of a spin-signature from a single Ho adatom as probed by spin-sensitive tunneling. Nature Communications, 7:10454, February 2016.

[106] J. Tersoff and D. R. Hamann. Theory and application for the scanning tunneling microscope. Phys. Rev. Lett., 50:1998-2001, Jun 1983.

[107] D. Wortmann, S. Heinze, Ph. Kurz, G. Bihlmayer, and S. Blügel. Resolving complex atomic-scale spin structures by spin-polarized scanning tunneling microscopy. Phys. Rev. Lett., 86:4132-4135, Apr 2001.

[108] Simon Holzberger, Tobias Schuh, Stefan Blügel, Samir Lounis, and Wulf Wulfhekel. Parity effect in the ground state localization of antiferromagnetic chains coupled to a ferromagnet. Phys. Rev. Lett., 110:157206, Apr 2013. 
[109] S. Baumann, F. Donati, S. Stepanow, S. Rusponi, W. Paul, S. Gangopadhyay, I. G. Rau, G. E. Pacchioni, L. Gragnaniello, M. Pivetta, J. Dreiser, C. Piamonteze, C. P. Lutz, R. M. Macfarlane, B. A. Jones, P. Gambardella, A. J. Heinrich, and H. Brune. Origin of perpendicular magnetic anisotropy and large orbital moment in fe atoms on mgo. Phys. Rev. Lett., 115:237202, Dec 2015.

[110] Feliciano Giustino. Electron-phonon interactions from first principles. Rev. Mod. Phys., 89:015003, Feb 2017.

[111] A. Eiguren, B. Hellsing, F. Reinert, G. Nicolay, E. V. Chulkov, V. M. Silkin, S. Hüfner, and P. M. Echenique. Role of bulk and surface phonons in the decay of metal surface states. Phys. Rev. Lett., 88:066805, Jan 2002.

[112] Sebastian Loth, Susanne Baumann, Christopher P. Lutz, D. M. Eigler, and Andreas J. Heinrich. Bistability in Atomic-Scale Antiferromagnets. Science, 335(6065):196-199, January 2012.

[113] J. Hermenau, J. Ibañez-Azpiroz, Chr Hübner, A. Sonntag, B. Baxevanis, K. T. Ton, M. Steinbrecher, A. A. Khajetoorians, M. dos Santos Dias, S. Blügel, R. Wiesendanger, S. Lounis, and J. Wiebe. A gateway towards non-collinear spin processing using three-atom magnets with strong substrate coupling. Nature Communications, 8(1):642, 2017.

[114] Daniel Canet and Pierre Mutzenhardt. Relaxation in Nuclear Magnetic Resonance, General. In Encyclopedia of Analytical Chemistry. John Wiley \& Sons, Ltd, 2006.

[115] Philip Willke, William Paul, Fabian D. Natterer, Kai Yang, Yujeong Bae, Taeyoung Choi, Joaquin Fernández-Rossier, Andreas J. Heinrich, and Christoper P. Lutz. Probing quantum coherence in single-atom electron spin resonance. Science Advances, 4(2), 2018.

[116] V. Schendel, C. Barreteau, M. Brandbyge, B. Borca, I. Pentegov, U. Schlickum, M. Ternes, P. Wahl, and K. Kern. Strong paramagnon scattering in single atom pd contacts. Phys. Rev. B, 96:035155, Jul 2017. 